\title{
Asymptotic expansion of velocity field within the front of viscoplastic surges: comparison with experiments
}

\author{
G. Chambon ${ }^{1} \dagger$, Perrine Freydier ${ }^{2,1}$, M. Naaim ${ }^{1}$ and J.-P. Vila ${ }^{3}$ \\ ${ }^{1}$ Univ. Grenoble Alpes, IRSTEA, UR ETGR, F-38402 Saint-Martin-d'Hères, France \\ ${ }^{2}$ Laboratoire FAST, Univ. Paris Sud, CNRS, Univ. Paris-Saclay, F-91405 Orsay, France \\ ${ }^{3}$ Institut de Mathématiques de Toulouse, UMR 5219, Univ. Toulouse, CNRS, INSA, F-31077 \\ Toulouse, France
}

(Received xx; revised xx; accepted $\mathrm{xx}$ )

This paper investigates the internal dynamics (velocity profiles and shear rate) of freesurface surges made of viscoplastic fluids. Compared to fluids without a yield stress, additional complexity arises from the possible coexistence of sheared and unsheared (or pseudo-plug) zones in the flow. Expanding on the thin-layer approach of Fernandez-Nieto et al. (2010), we derive formal asymptotic expansions of the velocity field and discharge up to $O(\epsilon)$ with respect to flow aspect ratio $\epsilon$. Detailed comparisons between these theoretical predictions and experimental data reveal that, while the leading-order approximation (equivalent to a lubrication model) satisfactorily accounts for the global dynamics of the flow, considering $O(\epsilon)$ correction terms is required to capture the evolution of velocity and shear rate close to the tip. Notably, these correction terms are responsible for the vanishing of the unsheared layer in the tip region, a feature clearly observed in the experiments. Differences between the leading-order and $O(\epsilon)$ models appear to be enhanced by the viscoplastic character of the fluid. In particular, $O(\epsilon)$ correction terms related to the existence of $O(1)$ plastic normal stresses in the pseudo-plug layer, play a critical role. This study provides important insights for future development of consistent shallow-water models adapted to viscoplastic materials.

\section{Introduction}

Since the pioneering work of Saint-Venant (1871), the modelling of free-surface flows via thin-layer approaches has been the subject of countless studies in hydraulics and fluid mechanics (e.g., Whitham 1974; Chanson 2004; Kalliadasis et al. 2012). In these models, the characteristic flow thickness $H$ is assumed to be small compared to the characteristic flow length $L$, i.e. flow aspect ratio $\epsilon=H / L \ll 1$. This includes lubrication models, in which inertia terms are neglected, as well as more general shallow-water (or Saint-Venantlike) models. Lubrication models typically reduce to a single advection-diffusion equation for the evolution of flow height $h$ (Benney 1966; Hunt 1994; Huppert 2006; Ancey et al. 2009). Shallow-water models are generally formulated as systems of two coupled equations (in 2D) for flow height $h$ and discharge $q$ or depth-averaged velocity $\bar{u}=q / h$ (Savage \& Hutter 1989; Ancey et al. 2007; Iverson 2013; Noble \& Vila 2013), although recently more robust three-equation models, including an additional enstrophy variable, have also been proposed (Richard et al. 2016). In these models, fluid rheology is accounted for through closure terms that implicitly depend on the distribution of longitudinal velocity $u(y)$

$\dagger$ Email address for correspondence: guillaume.chambon@irstea.fr 
(with $y$ the cross-stream coordinate) across the flowing layer (Ng \& Mei 1994; RuyerQuil \& Manneville 2000; Hogg \& Pritchard 2004). Most of the above-mentioned references considered the case of Newtonian or generalised Newtonian (e.g., power-law) fluids, either in laminar or turbulent regimes. In this work, we focus on a more complex class of fluids, namely viscoplastic fluids characterised by a yield stress (Balmforth et al. 2014; Coussot 2014). These materials are involved in numerous industrial (Roussel \& Coussot 2005; Frigaard et al. 2017) and geophysical applications (Laigle \& Coussot 1997; Ancey 2007), for which accurate thin-layer models would be needed. Yet, as will be discussed, the viscoplastic rheology poses specific modelling issues related to the possible coexistence of unyielded (solid-like) and yielded (fluid-like) zones within the flows. Hence, attempts at developing lubrication (Huang \& Garcia 1998; Balmforth et al. 2006; Ancey \& Cochard 2009; Bernabeu et al. 2014; Liu et al. 2016) or shallow-water (Coussot 1994; Liu \& Mei 1994; Piau 1996; Balmforth \& Liu 2004) models for viscoplastic materials remained relatively rare.

Formally, thin-layer models can be derived from the primitive Cauchy equations through asymptotic expansions with respect to flow aspect ratio $\epsilon$. Lubrication models proceed from a leading-order expansion (Benney 1966; Ruyer-Quil \& Manneville 1998). At the next level of approximation, derivation of consistent shallow-water models requires expansion of the velocity profile $u(y)$ up to at least $O(\epsilon)$ terms (Ruyer-Quil \& Manneville 2000; Noble \& Vila 2013). Yet, most shallow-water models used in practical applications, either for Newtonian of non-Newtonian fluids, are based on simpler, ad-hoc assumptions. Typically, closure terms are postulated empirically, or the velocity profile is assumed identical to that obtained in a steady uniform regime (see, e.g., Liu \& Mei 1994; Coussot 1994, for viscoplastic fluids). Such ad-hoc shallow-water models generally fail to predict the correct instability threshold for the flows (Ruyer-Quil \& Manneville 2000; Kalliadasis et al. 2012). From an operational point of view, these models nevertheless proved capable of producing relatively accurate predictions in terms of, e.g., front propagation velocity or free-surface shape (Hogg \& Pritchard 2004; Ancey et al. 2012; Saingier et al. 2016; Kirstetter et al. 2016).

Expansions of the velocity field up to $O(\epsilon)$, and the use thereof in the formulation of consistent shallow-water models, have been worked out for Newtonian and powerlaw fluids. The handling of the two types of constitutive laws is largely similar, despite a singularity in the apparent viscosity at the free surface in the power-law case. Ruyer-Quil \& Manneville (2000) and Ruyer-Quil et al. (2012) used a weighted-residual method to express deviations from the steady uniform profile in terms of polynomial test functions (see also Amaouche et al. 2012). Alternatively, Fernandez-Nieto et al. (2010) and Noble \& Vila (2013) performed a formal asymptotic expansion of the primitive equations, in a regime where these equations reduce to steady uniform flow at leading-order. At $O(\epsilon)$, the velocity profile $u(y)$ then explicitly depends on flow height gradient $\partial_{x} h$ (with $x$ the streamwise coordinate), and expresses as a sum of the steady uniform profile and of inertia correction terms. Shallow-water models derived from either of these two approaches were shown to correctly capture the flow instability threshold. More generally, Boutounet et al. (2016) explained that a whole family of consistent shallow-water models, which are all equivalent at $O(\epsilon)$, can be constructed. For practical applications, additional model selection criteria would thus be needed.

Far fewer studies focused on expansions of the velocity field in viscoplastic fluids described by Bingham or Herschel-Bulkley constitutive laws. In a steady uniform regime, the longitudinal velocity profile $u(y)$ is composed of a yielded layer at the base, overlain by an unyielded, plug layer close to the free-surface (e.g., Coussot 1994; Chambon et al. 2009). Balmforth \& Craster (1999) showed that, in thin non-uniform flows, this plug layer 
becomes a pseudo-plug in which the strain rate is $O(\epsilon)$. These authors worked out an asymptotic expansion of the velocity profile in the pseudo-plug up to $O(\epsilon)$ in the inertialess limit. Recently, Liu et al. (2019) investigated the transition between a true plug and a pseudo-plug. Balmforth \& Liu (2004) used the weighted-residual approach to derive an $O(\epsilon)$ velocity approximation in the sheared layer, but did not consider the correction terms in the pseudo-plug. Fernandez-Nieto et al. (2010) applied their formal asymptotic approach to the case of a Bingham fluid and showed that, besides the inertia correction terms already present for fluids without a yield stress, the $O(\epsilon)$ velocity approximation also involves correction terms due to the existence of $O(1)$ normal stresses in the pseudoplug. These latter authors also proposed the - to date - only consistent shallow-water model for a viscoplastic rheology. However, variations of pseudo-plug thickness along the flow are not considered in this model. Lubrication-like models, on the contrary, predict a dependence of pseudo-plug thickness on flow height gradient $\partial_{x} h$ (Balmforth \& Craster 1999; Ancey \& Cochard 2009; Freydier et al. 2017), and one would like $O(\epsilon)$ models to recover this feature when inertia terms are neglected.

In this context, the objectives of the present study are twofold. First, expanding on the approach of Fernandez-Nieto et al. (2010), we derive a complete $O(\epsilon)$ expansion of longitudinal velocity profile $u(y)$ in a Herschel-Bulkley thin free-surface flow, accounting for possible variations in pseudo-plug thickness. As will be shown, the expression obtained depends on gradients of both flow height and pseudo-plug thickness. The prospect of formulating consistent shallow-water models based on this asymptotic expansion of velocity, is left for future work. Instead, we assess the differences between the leadingorder and $O(\epsilon)$ velocity approximations in the specific case of travelling-wave solutions, for which the evolution of free surface can be computed without additional developments. The second objective is then to compare these asymptotic velocity predictions to highresolution experimental data recently obtained by our group (Freydier et al. 2017). Our previous works showed in particular that, in viscoplastic free-surface surges, longitudinal velocity profiles measured close to the tip clearly diverge from the predictions of a leading-order lubrication model. Our goal here is therefore to investigate whether an $O(\epsilon)$ approximation provides a better match to the data.

Formal asymptotic expansion of the velocity field is derived in section 2 . Section 3 examines the influence of the different correction terms involved in the $O(\epsilon)$ approximation, as a function of the relevant non-dimensional parameters. Experimental results are briefly recalled, and compared to the theoretical predictions, in section 4 . Finally, sections 5 and 6 discuss and summarise the main outcomes of the study. Additional results obtained with Newtonian fluids are presented in Appendix B.

\section{Thin-layer asymptotic expansion}

Let us consider a free-surface viscoplastic surge propagating down an inclined plane forming an angle $\theta$ to the horizontal (figure 1 ). The flow is driven by gravity $\boldsymbol{g}$. The $x$-axis (hereafter called longitudinal) coincides with the base plane in the streamwise direction, while the $y$-axis (hereafter called vertical) is orthogonal to the bed, pointing upwards. Flow is assumed to be 2D, i.e. independent of the spanwise direction. Fluid density is denoted $\rho$, local flow thickness $h(x, t)$, and fluid velocity $\boldsymbol{u}(x, y, t)$.

The viscoplastic fluid is assumed to obey Herschel-Bulkley constitutive law:

$$
\begin{cases}\tau_{i j}=\left(\frac{\tau_{c}}{|\dot{\gamma}|}+K|\dot{\gamma}|^{n-1}\right) \dot{\gamma}_{i j} & \text { if }|\tau|>\tau_{c} \\ \dot{\gamma}_{i j}=0 & \text { if }|\tau| \leqslant \tau_{c}\end{cases}
$$




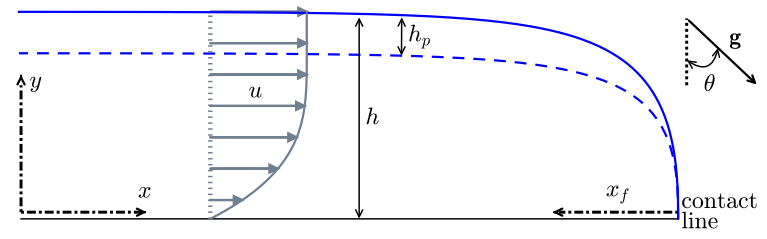

FiguRE 1. Free-surface viscoplastic surge down an incline: flow configuration considered in this study (see notations in text). Dashed line represents the fake yield surface.

where $\tau_{i j}=\sigma_{i j}+p \delta_{i j}$ is the extra-stress tensor (with $\sigma_{i j}$ the total stress and $p$ the pressure) and $\dot{\gamma}_{i j}=\partial_{j} u_{i}+\partial_{j} u_{i}$ is the strain-rate tensor. The tensorial norm is defined as $|\boldsymbol{T}|=\left(0.5 T_{i j} T_{i j}\right)^{0.5}$ for any second-order tensor $\boldsymbol{T}$. Rheological parameters $\tau_{c}, K$, and $n$ correspond to the yield stress, the consistency, and the power index of the material, respectively. We assume $n>0$, and shall consider more specifically the two cases $n=1$ (Bingham fluid) and $n \approx 0.4$ (shear-thinning value representative of most real viscoplastic fluids). In the following, for $|\tau|>\tau_{c}$, we split the extra-stress tensor into its viscous and plastic contributions: $\tau_{i j}=\tau_{i j}^{v}+\tau_{i j}^{p}$, with $\tau_{i j}^{v}=K|\dot{\gamma}|^{n-1} \dot{\gamma}_{i j}$ and $\tau_{i j}^{p}=\left(\tau_{c} /|\dot{\gamma}|\right) \dot{\gamma}_{i j}$.

\subsection{Primitive equations}

Let us denote $u=u_{x}$ and $v=u_{y}$ the longitudinal and vertical velocity components, respectively. The primitive equations governing free-surface flows of viscoplastic fluids are derived in numerous previous references (e.g., Piau 1996; Ancey \& Cochard 2009; Fernandez-Nieto et al. 2010). Here, we recall them directly in dimensionless form. Hence let us define the Reynolds, Froude and Bingham numbers as:

$$
R e=\frac{\rho U^{2-n} H^{n}}{K}, \quad F r=\frac{U}{\sqrt{g H \cos \theta}}, \quad B i=\frac{\tau_{c}}{K}\left(\frac{H}{U}\right)^{n},
$$

where $H, L$ and $U$ represent a characteristic thickness, a characteristic length, and a characteristic longitudinal velocity of the flow, respectively. Pressure $p$ is scaled by $\rho g H \cos \theta$, and shear rate $|\dot{\gamma}|$ is scaled by $U / H$. We choose to scale the viscous contribution to stresses by $K(U / H)^{n}$, and the plastic contribution by $\tau_{c}$. Lastly, the stress norm $|\tau|$ is scaled by $K(U / H)^{n}$, such that dimensionless yield condition writes $|\tau|=B i$.

Dimensionless mass and momentum conservation equations then read:

$$
\begin{aligned}
\partial_{x} u+\partial_{y} v & =0 \\
\epsilon \operatorname{Re}\left(\partial_{t} u+u \partial_{x} u+v \partial_{y} u\right) & =-\frac{\epsilon R e}{F^{2}} \partial_{x} p+\lambda+\partial_{y} \tau_{x y}^{v}+B i \partial_{y} \tau_{x y}^{p}+\epsilon\left(\partial_{x} \tau_{x x}^{v}+B i \partial_{x} \tau_{x x}^{p}\right) \\
\epsilon^{3} \operatorname{Re}\left(\partial_{t} v+u \partial_{x} v+v \partial_{y} v\right) & =-\frac{\epsilon R e}{F^{2}}\left(1+\partial_{y} p\right)+\epsilon^{2}\left(\partial_{x} \tau_{x y}^{v}+B i \partial_{x} \tau_{x y}^{p}\right)+\epsilon\left(\partial_{y} \tau_{y y}^{v}+B i \partial_{y} \tau_{y y}^{p}\right)
\end{aligned}
$$

where we recall that $\epsilon=H / L$ is flow aspect ratio, and the driving parameter $\lambda$ is given by:

$$
\lambda=\frac{\rho g H^{n+1} \sin \theta}{K U^{n}}=\frac{R e}{F r^{2}} \tan \theta .
$$


The dimensionless expressions of viscous and plastic stresses write, for $|\tau|>B i$ :

$$
\begin{aligned}
\tau_{x x}^{v}=-\tau_{y y}^{v} & =2 \epsilon|\dot{\gamma}|^{n-1} \partial_{x} u \\
\tau_{x y}^{v} & =|\dot{\gamma}|^{n-1}\left(\partial_{y} u+\epsilon^{2} \partial_{x} v\right),
\end{aligned}
$$

and

$$
\begin{aligned}
\tau_{x x}^{p}=-\tau_{y y}^{p} & =\frac{2 \epsilon}{|\dot{\gamma}|} \partial_{x} u \\
\tau_{x y}^{p} & =\frac{1}{|\dot{\gamma}|}\left(\partial_{y} u+\epsilon^{2} \partial_{x} v\right) .
\end{aligned}
$$

The equality $\tau_{x x}=-\tau_{y y}$ ensues from mass conservation (2.3). Lastly, the dimensionless stress norm expresses as:

$$
|\tau|=\sqrt{\left(\tau_{x y}^{v}+B i \tau_{x y}^{p}\right)^{2}+\left(\epsilon \tau_{x x}^{v}+B i \tau_{x x}^{p}\right)^{2}},
$$

and the dimensionless shear rate:

$$
|\dot{\gamma}|=\sqrt{\left(\partial_{y} u+\epsilon^{2} \partial_{x} v\right)^{2}+4 \epsilon^{2}\left(\partial_{x} u\right)^{2}}
$$

The above balance equations are to be complemented with appropriate boundary conditions. At the base we consider a classical no-penetration and no-slip condition: $u_{\mid y=0}=v_{\mid y=0}=0$. At the free surface, influence of surface tension is neglected and a stress-free condition is assumed: $(\boldsymbol{\tau}-p \boldsymbol{I})_{\mid y=h} \cdot \boldsymbol{n}=0$, with $\boldsymbol{n}=\left(-\partial_{x} h, 1\right)$ and $\boldsymbol{I}$ the identity tensor. Rearranging terms, we obtain:

$$
\begin{aligned}
\left(\tau_{x y}^{v}+B i \tau_{x y}^{p}\right)_{\mid y=h} & =\frac{2 \epsilon \partial_{x} h}{1-\epsilon^{2}\left(\partial_{x} h\right)^{2}}\left(\epsilon \tau_{x x}^{v}+B i \tau_{x x}^{p}\right)_{\mid y=h} \\
p_{\mid y=h} & =-\frac{F r^{2}}{R e} \frac{1+\epsilon^{2}\left(\partial_{x} h\right)^{2}}{1-\epsilon^{2}\left(\partial_{x} h\right)^{2}}\left(\epsilon \tau_{x x}^{v}+B i \tau_{x x}^{p}\right)_{\mid y=h} .
\end{aligned}
$$

Finally, the following kinematic condition holds at the free surface:

$$
\partial_{t} h+u_{\mid y=h} \partial_{x} h=v_{\mid y=h} .
$$

For completeness, we also recall the depth-averaged mass-conservation equation:

$$
\partial_{t} h+\partial_{x} q=0 \text {. }
$$

where $q=\int_{0}^{h} u d y$ is flow discharge. This equation is readily obtained from integration of local mass conservation (2.3) and boundary conditions.

\subsection{Flow regime}

Following the approach of Fernandez-Nieto et al. (2010), we aim to derive approximations of the velocity field in the flow through formal asymptotic expansions of the previous primitive equations with respect to $\epsilon$. The following assumptions are introduced: (i) $\epsilon \ll 1$ (thin-layer approximation). (ii) The factor $\epsilon R e$ in front of inertia terms in (2.4) is considered $O(\epsilon)$. (iii) As the study concerns flows in which plastic effects are significant, Bingham number $B i$ is considered $O(1)$. (iv) Finally, the factor $\epsilon R e / \mathrm{Fr}^{2}$ in front of pressure gradient terms in (2.4)-(2.5) is also considered $O(1)$. This latter assumption constitutes the main difference with the work of Fernandez-Nieto et al. (2010), in which $\epsilon R e / \mathrm{Fr}^{2}$ was considered $O(\epsilon)$. Accordingly, the flow regime investigated in the present study corresponds, at leading order, to a balance between pressure gradient, gravity and shear stress effects, as in classical lubrication models. Note that 
the assumption $\epsilon \operatorname{Re} / \mathrm{Fr}^{2}=O(1)$ implicitly requires the slope angle $\theta$ to be small, so that $\lambda=\epsilon R e / F^{2}(\tan \theta / \epsilon)$ can also be $O(1)$.

We assume the existence of regular expansions of the form: $f=f^{(0)}+\epsilon f^{(1)}+\ldots$, for all variables of interest, namely longitudinal and vertical velocities $u$ and $v$, pressure $p$, and stress components $\tau_{i j}$. As will be shown, these expansions can effectively be formally worked out up to $O(\epsilon)$. Let us note, however, that the residues, whose complete evaluation lies outside the scope of this paper, are not necessarily $O\left(\epsilon^{2}\right)$ and may involve terms depending on non-integer powers $\epsilon^{a}$ (with $a>1$ ) (Fernandez-Nieto et al. 2010).

\subsection{Leading-order expansion}

At $O(1)$ with respect to $\epsilon$, integration of equations (2.4)-(2.5) leads to linear profiles for the shear stress and pressure:

$$
\begin{aligned}
\tau_{x y}^{v,(0)}+B i \tau_{x y}^{p,(0)} & =\Lambda(x, t)(h-y), \\
p^{(0)} & =(h-y),
\end{aligned}
$$

where we introduced

$$
\Lambda(x, t)=\lambda-\frac{\epsilon R e}{F r^{2}} \partial_{x} h=\lambda\left(1-\epsilon \cot \theta \partial_{x} h\right) .
$$

Moreover, Herschel-Bulkley law reduces to, for $|\tau|>B i$ :

$$
\tau_{x y}^{v,(0)}+B i \tau_{x y}^{p,(0)}=\operatorname{sgn}\left(\partial_{y} u^{(0)}\right)\left(\partial_{y} u^{(0)}\right)^{n}+B i,
$$

while $\tau_{x x}^{p,(0)}=\tau_{x x}^{v,(0)}=0$. From these leading-order expressions, we expect the existence of an unsheared layer, in which $|\dot{\gamma}|^{(0)}=\left|\partial_{y} u^{(0)}\right|=0$, for $y \geqslant h-h_{p}$. The thickness of this layer is given by:

$$
h_{p}=\frac{B i}{\Lambda}
$$

We may assume, without loss of generality, that $\partial_{y} u^{(0)} \geqslant 0$. Integration of $(2.17)$ then leads to the classical lubrication velocity profile:

$$
u^{(0)}= \begin{cases}u_{p l}^{(0)}\left(1-\zeta^{(n+1) / n}\right) & \text { if } y<h-h_{p} \\ u_{p l}^{(0)} & \text { if } y \geqslant h-h_{p},\end{cases}
$$

where $\zeta=1-y /\left(h-h_{p}\right)$ is a reduced vertical coordinate, and

$$
u_{p l}^{(0)}=\frac{n}{n+1} \Lambda^{1 / n}\left(h-h_{p}\right)^{(n+1) / n} .
$$

It shall be emphasised that $h_{p}, \Lambda$, and thus $u_{p l}^{(0)}$, are all functions of $x$ and $t$ through the term $\partial_{x} h$ in (2.19). If $\epsilon R e / F r^{2}=0$, expressions (2.22)-(2.23) reduce to the velocity profile obtained in a steady uniform regime, with a constant plug thickness $h_{p}=B i / \lambda$ (e.g., Chambon et al. 2014).

The leading-order approximation of discharge $q$ immediately proceeds from (2.22):

$$
q^{(0)}=\frac{n}{2 n+1} \Lambda^{1 / n}\left(h-h_{p}\right)^{(n+1) / n}\left(h+\frac{n}{n+1} h_{p}\right) .
$$

In the following (see $\S 2.5$ ), leading-order expressions of vertical velocity $v^{(0)}$ and derivatives $\partial_{x, t} u^{(0)}$ will also be required. These quantities are easily derived from (2.22) and mass conservation (2.3), and we shall not provide the full expressions in this paper (see Freydier et al. 2017, for expression of $\left.v^{(0)}\right)$. Let us nevertheless mention that, compared to the developments of Fernandez-Nieto et al. (2010), additional complexities arise here 
from terms proportional to the derivatives $\partial_{x, t} h_{p}$ and $\partial_{x, t} \Lambda$. These derivatives can be interchanged through the following relation:

$$
\partial_{x, t} h_{p}=-\frac{h_{p}}{\Lambda} \partial_{x, t} \Lambda
$$

\subsection{Pseudo-plug}

The dependence upon $x$ of $u_{p l}^{(0)}$ appears incompatible with a strictly zero shear rate $|\dot{\gamma}|$ for $y \geqslant h-h_{p}$, as would be expected in a "true" unyielded plug. As observed by Balmforth \& Craster (1999), this apparent paradox is overcome by noting that, since $\partial_{y} u^{(0)}=0$ in this layer, the expansion of longitudinal velocity takes the form: $u=$ $u_{p l}^{(0)}(x, t)+\epsilon u_{p l}^{(1)}(x, y, t)+\ldots$. Accordingly, the leading-order expression of shear rate $|\dot{\gamma}|$ becomes:

$$
|\dot{\gamma}|=\epsilon \sqrt{\left(\partial_{y} u_{p l}^{(1)}\right)^{2}+4\left(\partial_{x} u_{p l}^{(0)}\right)^{2}}+\ldots,
$$

such that the expansion of Herschel-Bulkley law also needs to be revisited. In particular, unlike for $y<h-h_{p}$, the plastic normal stress $\tau_{x x}^{p}$ involves an $O(1)$ contribution:

$$
\tau_{x x}^{p,(0)}=\frac{2 \partial_{x} u_{p l}^{(0)}}{\sqrt{\left(\partial_{y} u_{p l}^{(1)}\right)^{2}+4\left(\partial_{x} u_{p l}^{(0)}\right)^{2}}}
$$

while the leading-order expression of shear stress becomes:

$$
\tau_{x y}^{v,(0)}+B i \tau_{x y}^{p,(0)}=B i \tau_{x y}^{p,(0)}=B i \frac{\partial_{y} u_{p l}^{(1)}}{\sqrt{\left(\partial_{y} u_{p l}^{(1)}\right)^{2}+4\left(\partial_{x} u_{p l}^{(0)}\right)^{2}}} .
$$

It ensues that the stress norm expresses as:

$$
|\tau|=B i+O\left(\epsilon^{n}\right)
$$

where the residue $O\left(\epsilon^{n}\right)$ is necessarily positive. Hence, plastic normal stresses actually make up for the small values of shear stress, and are sufficient for the yield criterion to be slightly exceeded. The unsheared layer predicted by the leading-order velocity expansion, should thus rather be considered as a pseudo-plug, in which the shear rate is $O(\epsilon)$. Accordingly, the interface at $y=h-h_{p}$ should be regarded as a fake yield surface.

Combining equations (2.28) and (2.17) leads to:

$$
\partial_{y} u_{p l}^{(1)}=\frac{2(h-y) / h_{p}}{\sqrt{1-\left((h-y) / h_{p}\right)^{2}}}\left|\partial_{x} u_{p l}^{(0)}\right|,
$$

where (2.21) was used to express pseudo-plug thickness $h_{p}$. From (2.26), the shear rate in the pseudo-plug then expresses as:

$$
|\dot{\gamma}|=\epsilon \frac{2}{\sqrt{1-\left((h-y) / h_{p}\right)^{2}}}\left|\partial_{x} u_{p l}^{(0)}\right|+\ldots
$$

Finally, leading-order plastic normal stress in the pseudo-plug writes:

$$
\tau_{x x}^{p,(0)}=\sqrt{1-\left(\frac{h-y}{h_{p}}\right)^{2}} \operatorname{sgn}\left(\partial_{x} u_{p l}^{(0)}\right) .
$$

Note that this expression for $\tau_{x x}^{p,(0)}$ requires $\partial_{x} u_{p l}^{(0)} \neq 0$. If $\partial_{x} u_{p l}^{(0)}=0$, we are either in a 
true plug $(|\dot{\gamma}|=0)$, or the shear rate in the pseudo-plug vanishes at $O(\epsilon)$ and a leading term of higher order needs to be considered in the expansion of $|\dot{\gamma}|$ (Fernandez-Nieto et al. 2010). This latter case will not be considered in this study.

\section{5. $O(\epsilon)$ expansion}

At $O(\epsilon)$, velocity expansions in the pseudo-plug $\left(y \geqslant h-h_{p}\right)$ and in the sheared layer $\left(y<h-h_{p}\right)$ need to be treated separately.

\subsubsection{In the pseudo-plug $\left(y \geqslant h-h_{p}\right)$}

As seen in $\S 2.4$, the $O(\epsilon)$ velocity correction in the pseudo-plug directly derives from the leading-order approximation of shear stress. Integrating (2.30) between $y=h-h_{p}$ and $y>h-h_{p}$, and accounting for the expression of $\partial_{x} u_{p l}^{(0)}$, leads to:

$$
u_{p l}^{(1)}=B i \mathcal{U}_{N p l}(x, y, t)+u_{+}(x, t)
$$

where

$$
\begin{aligned}
\mathcal{U}_{N p l}(x, y, t)=2 \Lambda^{(1-n) / n}\left(h-h_{p}\right)^{1 / n} & \sqrt{1-\left(\frac{h-y}{h_{p}}\right)^{2}} \times \\
& {\left[\partial_{x} h+\frac{1}{n+1}\left(h+n h_{p}\right) \frac{\partial_{x} \Lambda}{\Lambda}\right] \operatorname{sgn}\left(\partial_{x} u_{p l}^{(0)}\right), }
\end{aligned}
$$

and $u_{+}(x, t)$ is a matching term (see $\left.\S 2.5 .3\right)$.

\subsubsection{In the sheared layer $\left(y<h-h_{p}\right)$}

Expansion at $O(\epsilon)$ of momentum balance equations (2.4) and (2.5) leads to:

$$
\begin{aligned}
\partial_{y}\left(\tau_{x y}^{v,(1)}+B i \tau_{x y}^{p,(1)}\right)= & \frac{\epsilon R e}{F r^{2}} \partial_{x} p^{(1)}-B i \partial_{x} \tau_{x x}^{p,(0)} \\
& +\operatorname{Re}\left(\partial_{t} u^{(0)}+u^{(0)} \partial_{x} u^{(0)}+v^{(0)} \partial_{y} u^{(0)}\right) \\
\frac{\epsilon R e}{F r^{2}} \partial_{y} p^{(1)} & =-B i \partial_{y} \tau_{x x}^{p,(0)}
\end{aligned}
$$

Accounting for boundary conditions at the free surface, integration of (2.36) provides:

$$
\frac{\epsilon R e}{F r^{2}} p^{(1)}=-B i \tau_{x x}^{p,(0)},
$$

for all values of $y$. Accordingly, $p^{(1)}=0$ for $y<h-h_{p}$. Equation (2.37) also shows that the two terms $\left(\epsilon R e / F r^{2}\right) \partial_{x} p^{(1)}$ and $-B i \partial_{x} \tau_{x x}^{p,(0)}$, involved in (2.35), are equal everywhere in the flow. Integration of (2.35) between $y<h-h_{p}$ and $y=h$ then leads to the expression of the $O(\epsilon)$ shear-stress correction in the sheared layer:

$$
\tau_{x y}^{v,(1)}+B i \tau_{x y}^{p,(1)}=-B i \frac{\pi}{2} \frac{h_{p}}{\Lambda} \operatorname{sgn}\left(\partial_{x} u_{p l}^{(0)}\right) \partial_{x} \Lambda+R e \mathcal{I}(x, y, t),
$$

with

$$
\mathcal{I}(x, y, t)=-\int_{y}^{h}\left(\partial_{t} u^{(0)}+u^{(0)} \partial_{x} u^{(0)}+v^{(0)} \partial_{y} u^{(0)}\right) d y .
$$

The first term on the r.h.s. of (2.38) arises from the calculation of

$$
2 B i \int_{h-h_{p}}^{h} \partial_{x} \tau_{x x}^{p,(0)} d y+\left(\tau_{x y}^{v,(1)}+B i \tau_{x y}^{p,(1)}\right)_{\mid y=h},
$$


where the integral of $\partial_{x} \tau_{x x}^{p,(0)}$ is restricted to the range $\left[h-h_{p}, h\right]$, since $\tau_{x x}^{p,(0)}=0$ for $y<h-h_{p}$, and is evaluated from (2.32).

Expansion of Herschel-Bulkley law at $O(\epsilon)$ for $y<h-h_{p}$ writes:

$$
\tau_{x y}^{v,(0)}+B i \tau_{x y}^{p,(0)}+\epsilon\left(\tau_{x y}^{v,(1)}+B i \tau_{x y}^{p,(1)}\right)=\left(\partial_{y} u^{(0)}+\epsilon \partial_{y} u^{(1)}\right)^{n}+B i,
$$

since $|\dot{\gamma}|^{(0)}+\epsilon|\dot{\gamma}|^{(1)}=\left|\partial_{y} u^{(0)}+\epsilon \partial_{y} u^{(1)}\right|$. We assumed, again, that $\partial_{y} u^{(0)}+\epsilon \partial_{y} u^{(1)} \geqslant 0$. A Taylor expansion then leads to:

$$
\partial_{y} u^{(1)}=\frac{1}{n}\left(\partial_{y} u^{(0)}\right)^{1-n}\left(\tau_{x y}^{v,(1)}+B i \tau_{x y}^{p,(1)}\right) .
$$

Finally, inserting equation (2.38) into (2.41) and further integrating, provides the $O(\epsilon)$ longitudinal-velocity correction for $y<h-h_{p}$ :

$$
u^{(1)}=B i \mathcal{U}_{N}(x, \zeta, t)+\operatorname{Re} \mathcal{U}_{I}(x, \zeta, t)
$$

where

$$
\begin{aligned}
\mathcal{U}_{N}(x, \zeta, t) & =-\frac{\pi}{2} \Lambda^{(1-2 n) / n} h_{p}\left(h-h_{p}\right)^{1 / n}\left(1-\zeta^{1 / n}\right) \operatorname{sgn}\left(\partial_{x} u_{p l}^{(0)}\right) \partial_{x} \Lambda \\
\mathcal{U}_{I}(x, \zeta, t) & =\frac{1}{n} \Lambda^{(1-n) / n}\left(h-h_{p}\right)^{1 / n} \int_{\zeta}^{1} \zeta^{\prime(1-n) / n} \mathcal{I}\left(\zeta^{\prime}\right) d \zeta^{\prime}
\end{aligned}
$$

and we recall that $\zeta=1-y /\left(h-h_{p}\right)$. Explicit calculations of the functions $\mathcal{I}$ and $\mathcal{U}_{I}$ are lengthy but straightforward. The final expression of $\mathcal{U}_{I}$ is given in (A 2), Appendix A. Note that in this expression, following Fernandez-Nieto et al. (2010), all factors proportional to $\partial_{t} h$ have been replaced through the use of differential identity (A 1$)$, which derives from mass conservation equation.

\subsubsection{Matching and final expressions}

The term $u_{+}$in (2.33) is determined by patching the solutions obtained in the sheared layer and the pseudo-plug, i.e. enforcing continuity of $u^{(1)}$ at the fake yield surface $y=$ $h-h_{p}($ or $\zeta=0)$ :

$$
u_{+}=B i \mathcal{U}_{N}(x, 0, t)+\operatorname{Re} \mathcal{U}_{I}(x, 0, t) .
$$

Finally, the $O(\epsilon)$ longitudinal-velocity correction can thus be written as follows:

$$
u^{(1)}= \begin{cases}B i \mathcal{U}_{N}(x, \zeta, t)+\operatorname{Re} \mathcal{U}_{I}(x, \zeta, t) & \text { if } y<h-h_{p} \\ B i\left[\mathcal{U}_{N p l}(x, y, t)+\mathcal{U}_{N}(x, 0, t)\right]+\operatorname{Re} \mathcal{U}_{I}(x, 0, t) & \text { if } y \geqslant h-h_{p}\end{cases}
$$

where the functions $\mathcal{U}_{N p l}, \mathcal{U}_{N}$, and $\mathcal{U}_{I}$ are given by (2.34), (2.43), and (A 2), respectively. Terms in factor of $B i$ in (2.46) represent $O(\epsilon)$ corrections arising from the existence of $O(1)$ plastic normal stresses in the pseudo-plug. These correction terms, denoted henceforth $u_{N}^{(1)}$, vanish in the absence of a yield stress, i.e. for $B i=0$. It can also be noted that the normal-stress correction term affecting the sheared layer, namely $B i \mathcal{U}_{N}$, is proportional to $\partial_{x} \Lambda$ and thus to gradient of pseudo-plug thickness $\partial_{x} h_{p}$. The term $\operatorname{Re} \mathcal{U}_{I}(\zeta)$ in $(2.46)$, denoted henceforth $u_{I}^{(1)}$, represents the $O(\epsilon)$ correction associated to inertia effects. This term is present even for fluids without a yield stress, although its expression is considerably simplified when $h_{p}=0$ (see Appendix B for the Newtonian case).

From (2.46), the $O(\epsilon)$ correction on flow discharge $q$ is readily obtained:

$$
q^{(1)}=B i \mathcal{Q}_{N}(x, t)+\operatorname{Re} \mathcal{Q}_{I}(x, t),
$$


where the normal-stress correction term $\mathcal{Q}_{N}$ writes:

$$
\mathcal{Q}_{N}(x, t)=\frac{\pi}{2} \Lambda^{(1-n) / n} h_{p}\left(h-h_{p}\right)^{1 / n} \operatorname{sgn}\left(\partial_{x} u_{p l}^{(0)}\right) \partial_{x} h,
$$

and expression of the inertia correction term $\mathcal{Q}_{I}$ is given in (A 5), Appendix A. Interestingly, it is observed that the factors proportional to $\partial_{x} \Lambda$ in $\mathcal{U}_{N}$ and $\mathcal{U}_{N p l}$ cancel out in the calculation of $\mathcal{Q}_{N}$, such that this latter function only involve a factor proportional to $\partial_{x} h$.

Finally, let us recall that the above expansions at $O(\epsilon)$ implicitly require that $\partial_{x} u_{p l}^{(0)} \neq 0$ everywhere in the flow. Accordingly, the sign factor $\operatorname{sgn}\left(\partial_{x} u_{p l}^{(0)}\right)$ involved in the normalstress correction terms $\mathcal{U}_{N p l}, \mathcal{U}_{N}$ and $\mathcal{Q}_{N}$, was assumed to remain constant along the flow.

\section{Influence of $O(\epsilon)$ correction terms for travelling-wave solutions}

This section aims to compare the leading-order and $O(\epsilon)$ asymptotic approximations established above, and to investigate the relative influences of the $O(\epsilon)$ normal-stress and inertia corrections terms. We explore configurations for which $B i$ and $R e$ numbers vary between $0.5-1.5$ and $0.1-1.5$, respectively, consistently with the ranges achieved in the experiments (see $\S 4.2$ ). A slope angle $\theta=15^{\circ}$ is considered, and results corresponding to power indices $n=1$ and $n=0.4$ are compared.

Expressions of $u^{(0)}$ and $u^{(1)}$ depend on flow thickness $h$, on its derivative $\partial_{x} h$, and, for $u^{(1)}$, on the second derivatives $\partial_{x x} h$ and $\partial_{x t} h$ (through $\partial_{x} \Lambda$ and $\partial_{t} \Lambda$ ). Quantitative evaluation of the asymptotic velocity profiles thus requires specifying a free-surface evolution. In practice, values of $h$ can be obtained either from experimental measurements or from a theoretical solution. We take here the latter approach, focusing on specific travelling-wave solutions of the form:

$$
h(x, t)=h_{t w}\left(x_{f}\right),
$$

with $x_{f}=u_{b} t-x$, and $u_{b}$ the wave propagation speed. For such solutions, mass conservation equation (2.16) implies that

$$
\frac{d}{d x_{f}}\left(u_{b} h-q\right)=0 .
$$

If, furthermore, $u_{b} h-q=0$ is assumed at surge tip, then the relation

$$
q=u_{b} h
$$

holds everywhere along the surge. These particular solutions are thus characterised by a constant depth-averaged velocity $\bar{u}=q / h=u_{b}$. Combining (3.2) with expressions (2.24) and (2.47) for the discharge $q$, closed-form ordinary differential equations (ODEs) for the free-surface shape $h_{t w}\left(x_{f}\right)$ can then be obtained (see below).

As will be shown, far from the tip, these travelling-wave solutions asymptotically converge to a steady uniform flow. To simplify expressions, we shall henceforth consider the following choice of characteristic scales: (i) Characteristic velocity $U$ is taken equal to the travelling-wave propagation speed $u_{b}$. (ii) Both characteristic thickness $H$ and characteristic length $L$ are taken equal to the flow thickness $H_{N}$ achieved in steady uniform regime for the average velocity $u_{b}$. Note that choosing identical vertical and longitudinal length scales formally amounts to considering $\epsilon=1$. Obviously, we remain however in the frame of a thin-layer assumption. With this particular choice of characteristic scales, 
and since $u_{b}$ and $H_{N}$ are related through the steady uniform condition, numbers $\lambda$ and $B i$ are linked as follows:

$$
\lambda=\left(\frac{2 n+1}{n}\right)^{n}\left(1-\frac{B i}{\lambda}\right)^{-(n+1)}\left(1+\frac{n}{n+1} \frac{B i}{\lambda}\right)^{-n} .
$$

\subsection{Free-surface shape}

In the following, two different free-surface models are considered. For computing leading-order velocity approximations, we define model $M_{1}$ based on the leading-order approximation of discharge $q$ :

$$
M_{1}: \quad q^{(0)}\left(h_{t w}, \frac{d h_{t w}}{d x_{f}}\right)=h_{t w}
$$

where the expression of $q^{(0)}$ as a function of $h_{t w}$ and $d h_{t w} / d x_{f}$ is provided by (2.24). Equation (3.4) constitutes a first-order ODE for the free-surface shape $h_{t w}\left(x_{f}\right)$. The boundary condition $h_{t w}\left(x_{f}=0\right)=0$ is enforced, such that $x_{f}$ represents the distance from surge tip. In practice, (3.4) is integrated numerically using python function odeint (see also Freydier et al. 2017). Due to the singularity of $d h_{t w} / d x_{f}$ at surge tip (see below), numerical integration is started from a small value $x_{f, 0}$, with the corresponding value $h_{t w}\left(x_{f, 0}\right)$ being obtained through a finite-difference approximation.

For computing velocity expansions at order $O(\epsilon)$, we consider model $M_{1+\epsilon}$ based on the $O(\epsilon)$ approximation of discharge:

$$
M_{1+\epsilon}: \quad q^{(0)}\left(h_{t w}, \frac{d h_{t w}}{d x_{f}}\right)+q^{(1)}\left(h_{t w}, \frac{d h_{t w}}{d x_{f}}, \frac{d^{2} h_{t w}}{d x_{f}^{2}}\right)=h_{t w},
$$

where the expression of $q^{(1)}$ is provided by (2.47). Equation (3.5) constitutes a secondorder ODE for $h_{t w}\left(x_{f}\right)$. It is solved by finite-difference discretisation on a regular mesh, using python function newton_krylov. The same boundary condition as for model $M_{1}$ is considered at $x_{f}=0$, and we impose $h_{t w} \approx 1$ sufficiently far from the tip (in practice, the value provided by model $M_{1}$ is taken). The iterations of the relaxation algorithm are initiated with the solution of model $M_{1}$.

Figure 2 shows comparisons between the free surfaces obtained with models $M_{1}$ and $M_{1+\epsilon}$ for different values of numbers $B i$ and $R e$. In all cases, the front is steep and perpendicular to channel bottom at the tip, namely $\left|\partial_{x} h\right| \rightarrow \infty$ as $x_{f} \rightarrow 0$. Values of the derivative $\left|\partial_{x} h\right|$ exceed 0.1 for $x_{f}<1.5-2$, typically, a position below which the validity of the thin-layer assumption may thus become questionable (see §5.2). Note however that values of $\left|\partial_{x} h\right|$ in excess of 1 are reached only very close to the tip, for $x_{f}<0.1$ typically. Away from the tip, the free surface monotonically increases towards the asymptotic value of 1 corresponding to a steady uniform regime. Flow height can be considered as essentially uniform for $x_{f}>10$, typically.

Globally, it appears that the free surfaces predicted by models $M_{1}$ and $M_{1+\epsilon}$ remain very close, regardless of the parameter values considered (figure 2). Note that model $M_{1}$ only depends on Bingham number $B i$, while model $M_{1+\epsilon}$ depends on both $B i$ and Re. For $n=0.4$ (figure 2a), and when $B i$ is small, the free surfaces obtained with the two models are hardly distinguishable. When $B i$ increases, small deviations between the different solutions become visible. It is observed that the free surfaces computed with model $M_{1+\epsilon}$ systematically lie below those obtained with model $M_{1}$. Moreover, for a given value of $B i$, the differences between the $M_{1}$ and the $M_{1+\epsilon}$ solutions are larger when $R e$ is small. Similar observations are made for $n=1$ (figure $2 \mathrm{~b}$ ).

Let us note that, far from the tip $\left(x_{f} \rightarrow \infty\right)$, expression (2.23) for the leading-order 

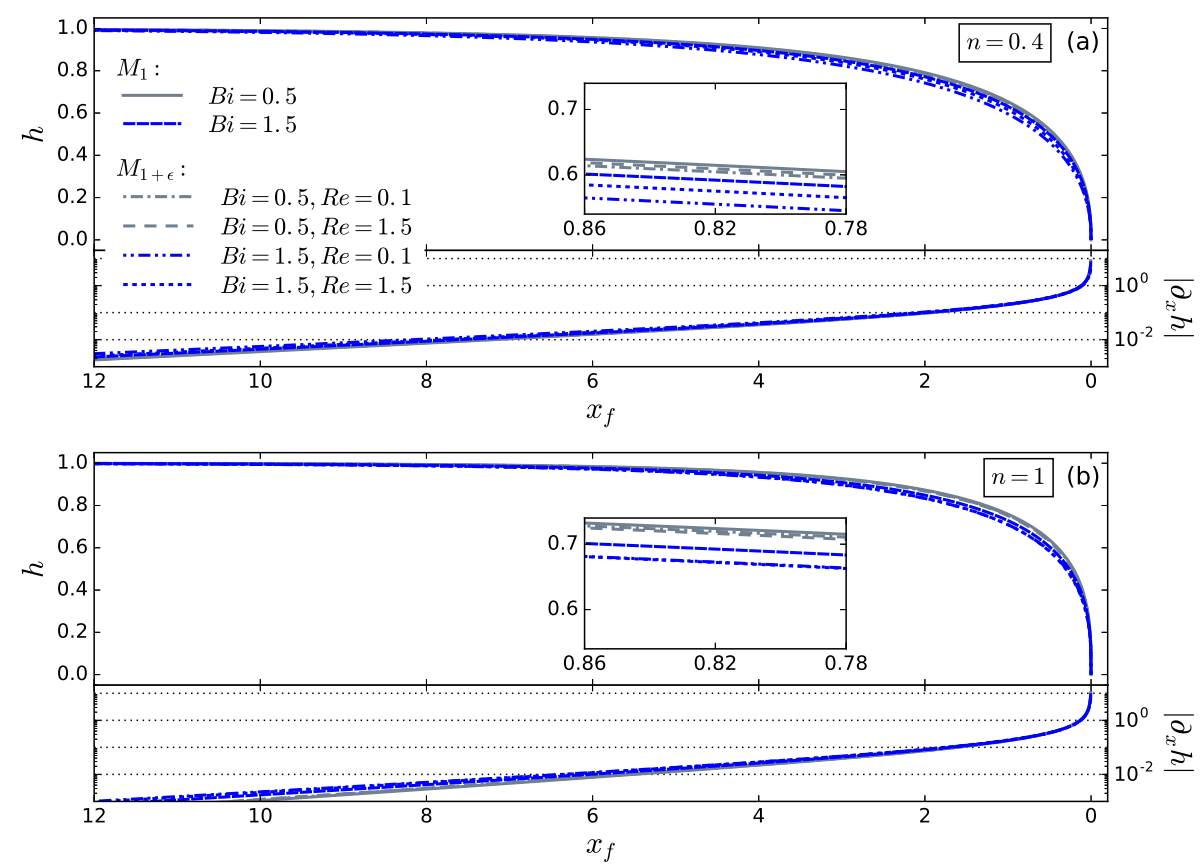

Figure 2. Free-surface shapes predicted by models $M_{1}$ and $M_{1+\epsilon}$ : evolution of flow height $h$ and derivative $\partial_{x} h$ versus distance from the tip $x_{f}$. (a) $n=0.4$. (b) $n=1$. In both plots, solutions computed for two values of $B i$ and two values of $R e$ are shown $\left(\theta=15^{\circ}\right)$. Insets show close-ups on the slight differences among the solutions.

velocity in the pseudo-plug can be approximated as:

$$
u_{p l}^{(0)} \approx \frac{n}{n+1} \lambda^{1 / n}\left(h-\frac{B i}{\lambda}\right)^{(n+1) / n} .
$$

Accordingly, $\operatorname{sgn}\left(\partial_{x} u_{p l}^{(0)}\right)=\operatorname{sgn}\left(\partial_{x} h\right)=-1$ for $x_{f} \rightarrow \infty$. Since, furthermore, the factor $\operatorname{sgn}\left(\partial_{x} u_{p l}^{(0)}\right)$ is assumed to be constant (see $\S 2.5 .3$ ), it is taken as uniformly equal to -1 along the flow for computing solutions of model $M_{1+\epsilon}$.

\subsection{Velocity profiles and free-surface velocity}

Approximations of the velocity profile $u(y)$ at leading-order and at $O(\epsilon)$ are computed from expressions (2.22) and (2.46). When limiting to leading order, free surfaces obtained from model $M_{1}$ are considered. Conversely, for computing velocity approximations at $O(\epsilon)$, free surfaces coming from model $M_{1+\epsilon}$ are taken into account. Considering these two, slightly-different, free-surface models ensures that all computed velocity profiles effectively comply with the condition $\bar{u}=1$. In the following, for convenience, the terminology $M_{1}$ and $M_{1+\epsilon}$ is also used for referring to the velocity profiles computed with either one of the two models. Again, the assumption $\operatorname{sgn}\left(\partial_{x} u_{p l}^{(0)}\right)=-1$ is made to evaluate the normal-stress correction terms. It was checked that the reconstructed $O(\epsilon)$ velocity profiles are effectively consistent with this assumption, namely that values of $u_{p l}^{(0)}$ calculated with model $M_{1+\epsilon}$ are decreasing towards the tip.

Figures $3 \mathrm{a}$ and $3 \mathrm{~b}$ present velocity profiles calculated for two values of $x_{f}$ and different values of $B i$ and $R e$. Only the case $n=0.4$ is shown here. Obviously, sufficiently far from the tip, velocities obtained with models $M_{1}$ and $M_{1+\epsilon}$ are essentially identical. At 

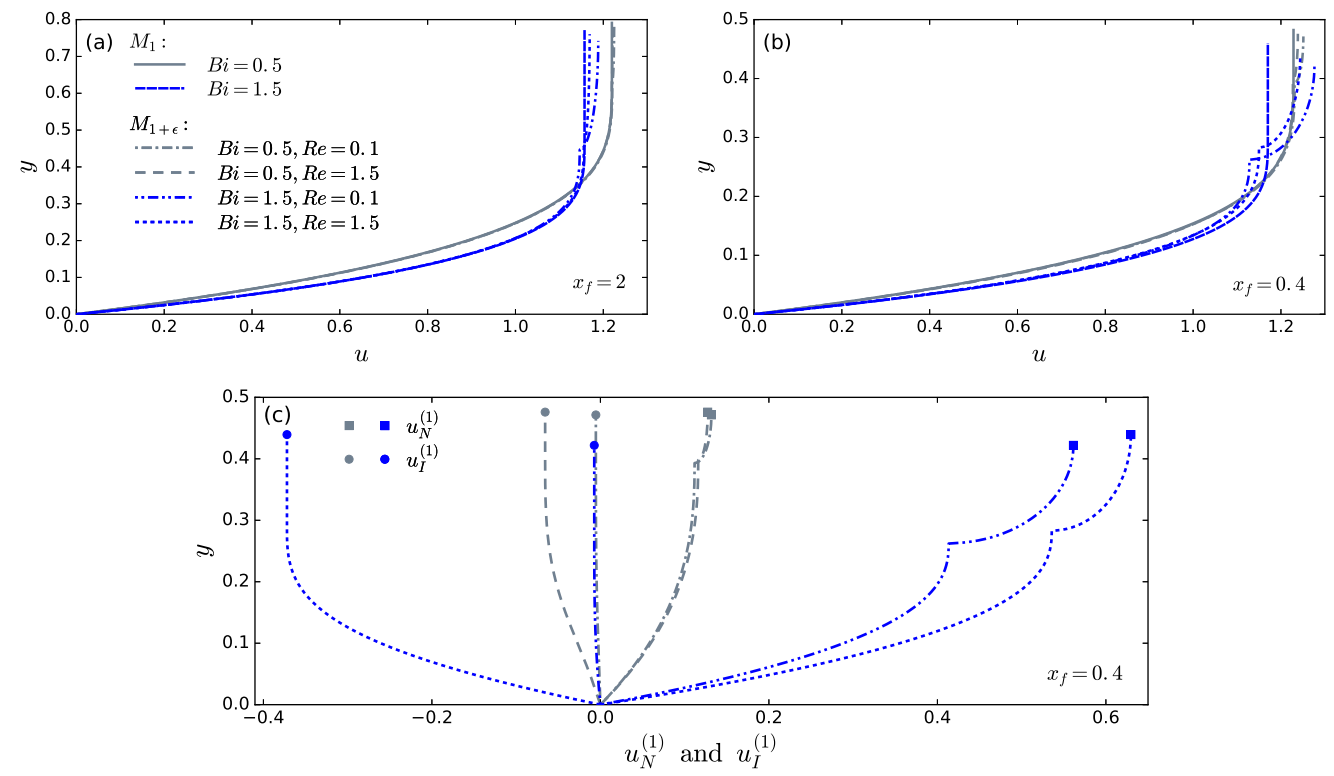

FIGURE 3. (a) and (b) Vertical profiles of longitudinal velocity $u$ computed with models $M_{1}$ and $M_{1+\epsilon}$ at two distances from the tip $x_{f}$ (see legend). In both plots, solutions computed for two values of $B i$ and two values of $R e$ are shown $\left(\theta=15^{\circ}, n=0.4\right)$. (c) Vertical profiles of the $O(\epsilon)$ normal-stress (thick lines with squares) and inertia (thin lines with circles) correction terms $u_{N}^{(1)}$ and $u_{I}^{(1)}$ at a position $x_{f}=0.4$.

$x_{f}=2$, slight differences between the predictions of the two models begin to appear for large values of $\mathrm{Bi}$ (figure 3a). These differences between the models are then magnified when moving closer to the tip, as shown in figure $3 \mathrm{~b}$ for $x_{f}=0.4$. Also clearly visible are the velocity gradients present in the pseudo-plug with model $M_{1+\epsilon}$. Furthermore, the corresponding velocity profiles present a nonphysical kink at $y=h-h_{p}$. As discussed below (see $\S 5.1$ ), this singularity arises from the patching of the $O(\epsilon)$ correction terms at the fake yield surface.

Globally, the interplay of numbers $B i$ and $R e$ on the velocity profiles is rather complex. Depending on the values of these parameters, and on the vertical position $y$, velocities predicted by model $M_{1+\epsilon}$ can either be larger or smaller than those predicted by model $M_{1}$. Figure $3 \mathrm{c}$ shows the typical relative magnitudes of the $O(\epsilon)$ normal-stress and inertia correction terms $u_{N}^{(1)}$ and $u_{I}^{(1)}$. These two correction terms generally display opposite signs (except for very low values of $B i$ ). The normal-stress term $u_{N}^{(1)}$ is positive and, as expected, increases with Bingham number $B i$. The inertia term $u_{I}^{(1)}$ is generally negative, and its absolute value increases with Reynolds number Re. More surprisingly, the inertia correction also appears to significantly increase (in absolute value) with $\mathrm{Bi}$. The counterbalancing effects between the two correction terms explain that, for a given value of $B i$, the net velocity correction tends to decrease when $R e$ increases (clearly visible in figure $3 \mathrm{~b}$ ). The net correction, however, systematically increases with $B i$ (as seen in figures $3 \mathrm{a}$ and $3 \mathrm{~b})$.

Figure 4 presents the evolution of free-surface velocity $u_{s}$ along the surge. For model $M_{1}, u_{s}=u_{p l}^{(0)}$, while for model $M_{1+\epsilon}, u_{s}=u_{p l}^{(0)}+\left.u_{p l}^{(1)}\right|_{y=h}$. Computed predictions have been truncated at $x_{f}=0.1$, i.e. at the position where $\left|\partial_{x} h\right|$ typically reaches a value of 1 (see figure 2). It was checked that, with model $M_{1+\epsilon}$, the asymptotic expansion 

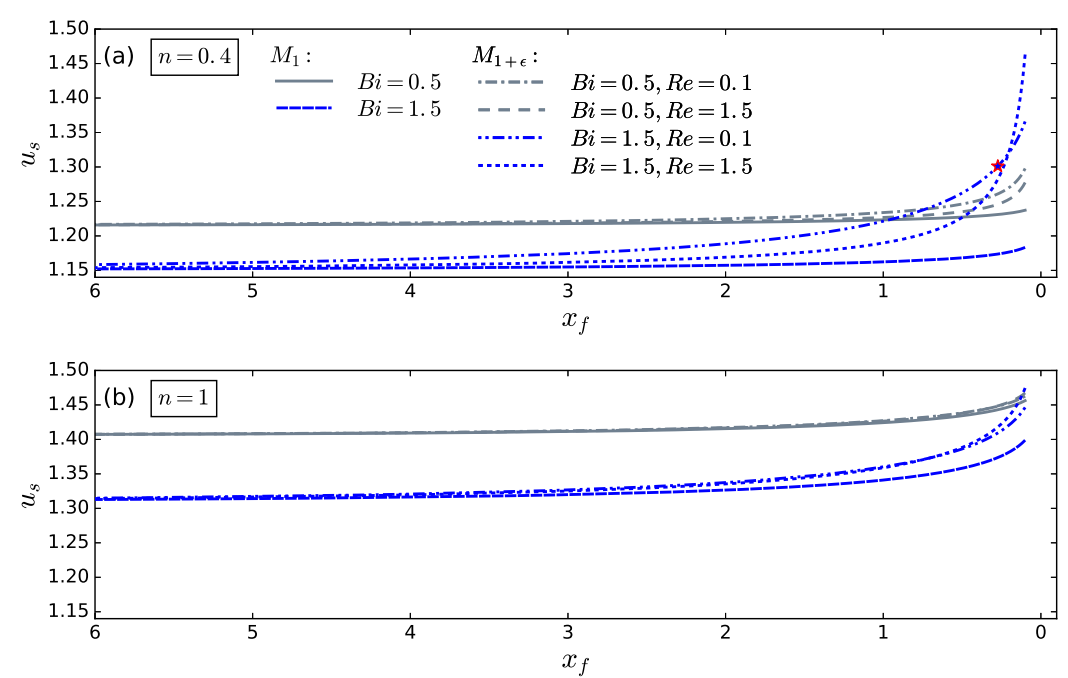

Figure 4 . Evolution of free-surface velocity $u_{s}$ predicted by models $M_{1}$ and $M_{1+\epsilon}$ as a function of distance from the tip $x_{f}$. (a) $n=0.4$. (b) $n=1$. In both plots, solutions computed for two values of $B i$ and two values of $R e$ are shown $\left(\theta=15^{\circ}\right)$. In plot (a), the red star marks the position where the asymptotic expansion in model $M_{1+\epsilon}$ ceases to be well-ordered for the case $B i=1.5, R e=0.1$ (blue dot-dashed line).

remains well-ordered, i.e. $u_{s}^{(1)}<u_{s}^{(0)}$, for $x_{f}>0.1$ in all but one of the displayed cases. With $n=0.4, B i=1.5$ and $R e=0.1$, the expansion ceases to be well-ordered at $x_{f}=0.25$, typically (red star in figure 4a). Globally, it is observed that, for the two models and all considered parameter values, surface velocity $u_{s}$ systematically increases towards the tip. Note that the increase of $u_{s}$ is not incompatible with the requirement that $\operatorname{sgn}\left(\partial_{x} u_{p l}^{(0)}\right)=-1$ in model $M_{1+\epsilon}$ (see $\left.\S 3.1\right)$. Again, the counterbalancing effects of the $O(\epsilon)$ normal-stress and inertia correction terms are clearly apparent for $n=0.4$ (figure 4a): The normal-stress correction tends to increase the value of $u_{s}$, while the inertia term generally acts in the opposite direction. The net correction, however, remains systematically positive: surface velocities obtained with model $M_{1+\epsilon}$ are larger than those obtained with model $M_{1}$, with differences becoming visible for $x_{f}<2-6$, typically. For similar values of $B i$ and $R e$, the influence of the $O(\epsilon)$ corrections, and notably of the inertia term, appear to be less marked in the case $n=1$ (figure $4 \mathrm{~b}$ ).

\subsection{Pseudo-plug shear rate and effective unsheared layer}

At $O(\epsilon)$, a strictly positive shear rate $|\dot{\gamma}|$ is predicted in the pseudo-plug (see $\S 2.4$ ). Integrating (2.31), the following expression is obtained for the $O(\epsilon)$ vertically-averaged shear rate $|\dot{\gamma}|_{p l}^{(1)}$ in the pseudo-plug:

$$
|\dot{\gamma}|_{p l}^{(1)}=\pi\left|\partial_{x} u_{p l}^{(0)}\right|
$$

As shown in figure 5, values of $|\dot{\gamma}|_{p l}^{(1)}$ obtained from model $M_{1+\epsilon}$ significantly depart from 0 for $x_{f}<2-6$, depending on the considered values of $B i$ and $R e$. Again, note that the plots are truncated at $x_{f}=0.1$. The increase in $|\dot{\gamma}|_{p l}^{(1)}$ towards the tip is primarily driven by the normal-stress correction, and is more pronounced for larger $B i$. Conversely, for $n=0.4$, the increase of $|\dot{\gamma}|_{p l}^{(1)}$ gets generally confined closer to the tip when $R e$ increases (counterbalancing effect, see figure 5a). For $n=1$, the net $O(\epsilon)$ correction 

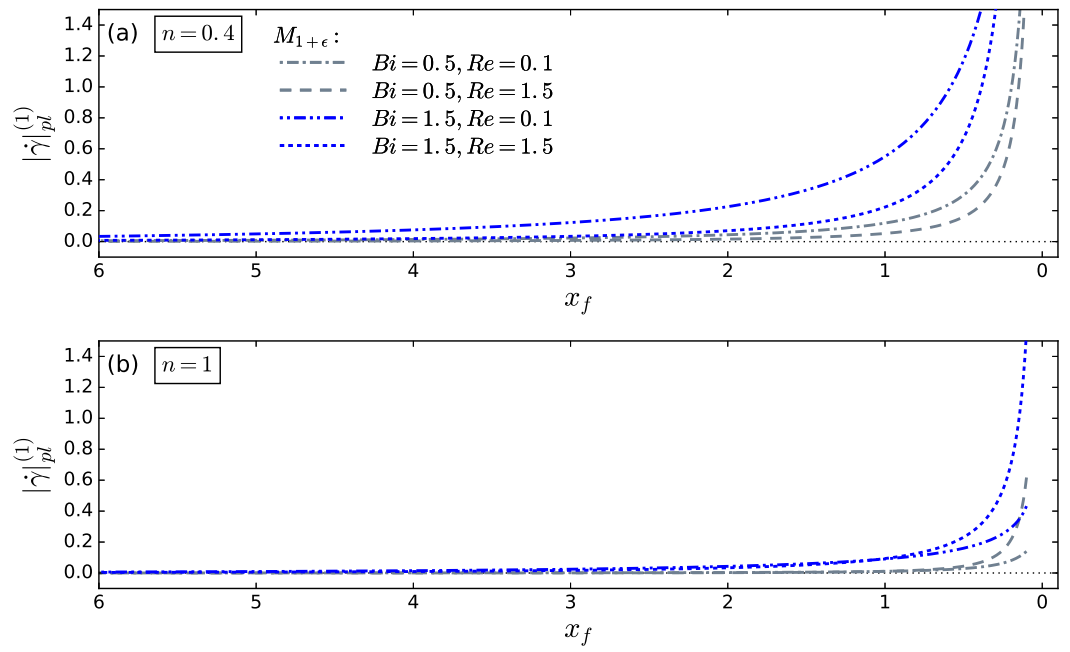

Figure 5. Evolution of $O(\epsilon)$ vertically-averaged shear rate in the pseudo-plug $|\dot{\gamma}|_{p l}^{(1)}$ predicted by model $M_{1+\epsilon}$, as a function of distance from the tip $x_{f}$. (a) $n=0.4$. (b) $n=1$. In both plots, solutions computed for two values of $B i$ and two values of $R e$ are shown $\left(\theta=15^{\circ}\right)$. The dotted line indicates the 0 value (i.e., $M_{1}$ solution).

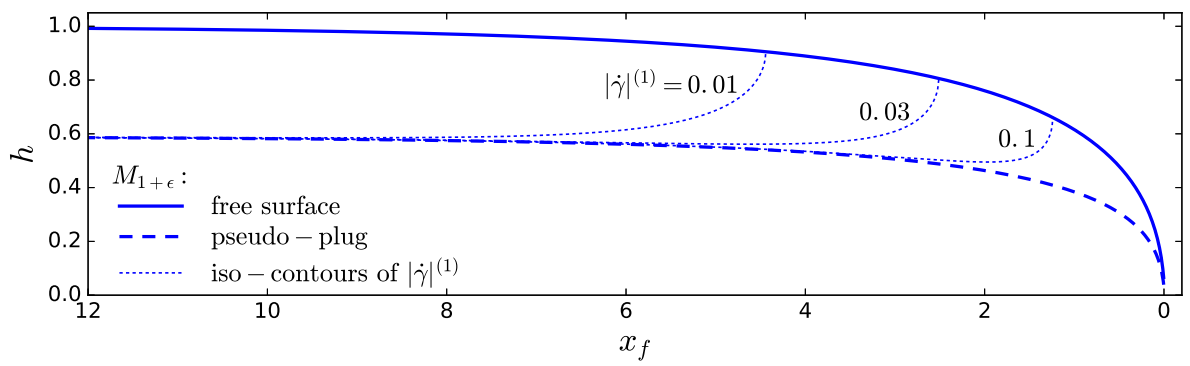

Figure 6. Iso-contours of $O(\epsilon)$ shear rate $|\dot{\gamma}|^{(1)}$ in the pseudo-plug predicted by model $M_{1+\epsilon}$ (dotted lines). The solid line represents the free-surface and the dashed line represents the fake yield surface $y=h-h_{p}\left(B i=1.5, R e=1.5, \theta=15^{\circ}, n=0.4\right)$.

is less pronounced, and the effect of $R e$ changes sign in the close vicinity of the tip (figure $5 b$ ).

Figure 6 displays a typical example of iso-contours of the $O(\epsilon)$ shear rate $|\dot{\gamma}|^{(1)}$ in the pseudo-plug. Recall that the pseudo-plug corresponds to an unsheared layer for the leading-order approximation. Similarly, one can define an effective unsheared layer at order $O(\epsilon)$, as the zone located above the particular $|\dot{\gamma}|^{(1)}$-contour corresponding to a chosen shear-rate threshold. For sufficiently large values of $x_{f}$, the boundary of this effective unsheared layer coincides with the fake yield surface $y=h-h_{p}$, regardless of the chosen threshold. When approaching the tip, however, the thickness of the effective unsheared layer decreases much faster than $h_{p}$, and abruptly collapses at a certain value of $x_{f}$. The precise position at which this collapse occurs, moves closer to the tip as the considered shear-rate threshold increases (figure 6). 

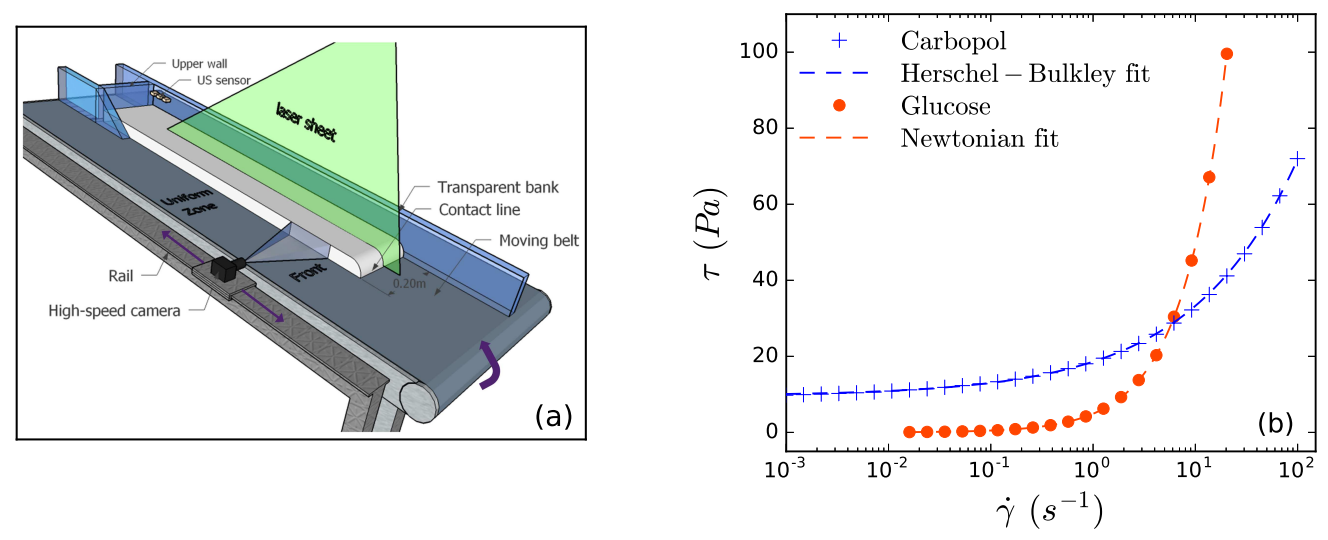

FiguRE 7. (a) Scheme of the conveyor-belt channel. The stationary surge is represented in white. (b) Typical steady-state flow curves of Carbopol and glucose samples used in the experiments: shear stress $\tau$ versus shear rate $\dot{\gamma}$ (semi-log scale). Symbols correspond to rheometrical measurements; dashed lines represent the best-fitting Herschel Bulkley and Newtonian laws (Carbopol: $\tau_{c}=9.6 \mathrm{~Pa}, K=9.0 \mathrm{~Pa} . \mathrm{s}^{n}, n=0.42$; Glucose: $\eta=5.6 \mathrm{~Pa} . \mathrm{s}$ ).

\section{Comparison with experimental results}

In this section, the leading-order and $O(\epsilon)$ velocity approximations are cross-compared to experimental results. The experimental dataset consists of high-resolution measurements of the internal velocity field within laboratory viscoplastic surges (Freydier et al. 2017). In parallel, free-surface shapes were also recorded. Experimental setup and methods were detailed in previous papers (Chambon et al. 2014; Freydier et al. 2017). Only the elements essential for the present comparison are recalled below.

\subsection{Review of experimental methods}

The experimental setup consists of a 3-m-long inclined channel, whose bottom is constituted by an upward-moving conveyor belt (figure 7a). Owing to this mobile bottom, gravity-driven free-surface surges that remain stationary in the laboratory reference frame, can be generated. When a given volume of fluid is placed in the channel, flow height spontaneously adapts to the fixed bottom velocity $u_{b}$ (Chambon et al. 2009, 2014). The studied surges have a typical length of $1 \mathrm{~m}$ and a typical height of $10-30 \mathrm{~mm}$, thereby complying with thin-layer assumption (see figure 8). Side-wall effects remain limited in this setup, such that transverse velocities are negligible and the flow can be regarded as effectively 2D along the centerline of the surges (except in the close vicinity of the tip: see Freydier et al. 2017).

Viscoplastic experiments were performed with Carbopol (EDT 2623). Even if it can display more complex rheological trends in transient flows, this polymeric micro-gel was used as a model viscoplastic fluid in numerous previous studies (e.g., Piau 2007; Bonn et al. 2017). As shown in figure 7b, the steady-state flow curve of the material is well represented by Herschel-Bulkley law. The yield stress $\tau_{c}$, consistency $K$, and power index $n$ of the samples were determined using a laboratory rheometer (Bohlin CVOR) equipped with roughened parallel-plate tools to avoid slip. Values of $n$ systematically lie around 0.4 (see table 1). Following our previous works (Chambon et al. 2014; Freydier et al. 2017), measured values of $\tau_{c}$ and $K$ were increased by $11 \%$ and $19 \%$, respectively, to ensure 


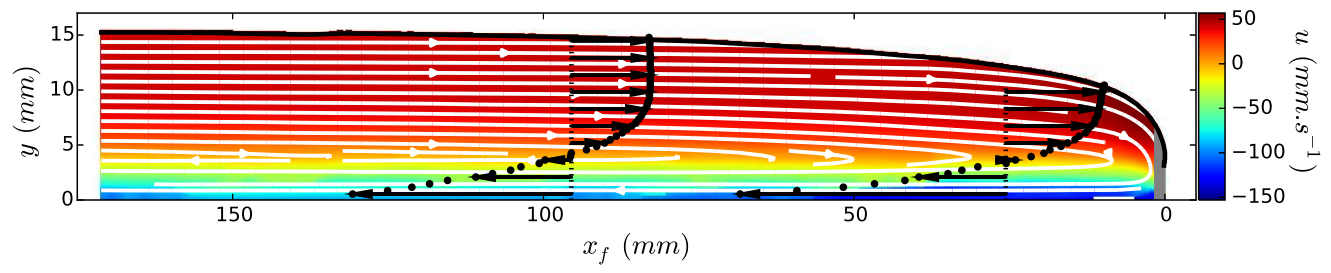

Figure 8. Measured longitudinal velocity $(u)$ field for experiment $\operatorname{HB} 13(B i=0.5, R e=1.4)$. The colour scale corresponds to raw velocity values (no shifting by bed velocity $u_{b}$ ). Streamlines are superimposed in white. Two vertical profiles of $u$, obtained at positions $x_{f}=95 \mathrm{~mm}$ and $x_{f}=25 \mathrm{~mm}$, respectively, are also represented. The grey area close to the tip indicates the zone where no reliable velocity measurements could be obtained.

quantitative agreement between predictions and measurements of flow height $H_{N}$ in the uniform zone of the surges, far from the tip.

A few benchmark experiments were also performed with a Newtonian fluid (glucose syrup). In this case, the viscosity $\eta$ of the samples was measured with a cone-plate geometry (figure $7 \mathrm{~b}$ ). Since glucose viscosity strongly depends on temperature (Schellart 2011), particular care was taken to perform experiments as rapidly as possible, and avoid temperature variations, when working with this material. Results obtained with Newtonian surges are summarised in Appendix B.

Flows are monitored by illuminating the centerplane of the surges with a laser sheet, and acquiring images from the side with a $500 \mathrm{~Hz}$ camera. The transparent fluids are seeded with $10 \mu \mathrm{m}$ tracers. We reconstruct velocity fields (see figure 8) through a spacetime analysis technique that takes full advantage of the stationary character of the surges (Freydier et al. 2017). This technique achieves higher spatial resolutions than more classical correlation methods (PIV), with typical values in our experiments of $1.24 \mathrm{~mm}$ in the longitudinal direction and $0.62 \mathrm{~mm}$ in the vertical direction In addition, local flow height and free-surface shape are also extracted from the images, with an uncertainty in the range $\pm 0.2-0.9 \mathrm{~mm}$.

An example of measured longitudinal velocity field is shown in figure 8. Far from the tip, the velocity profiles present the typical 2-layer shape expected in steady uniform regime, with an unsheared plug close to the free surface and a sheared layer underneath. When approaching the tip, velocity profiles begin to display shear throughout the entire depth of the flow. Accordingly, the unsheared zone seemingly disappears for $x_{f} / H_{N}<1-2$, typically (Freydier et al. 2017). Experimental measurements also feature an increase in surface velocity when approaching the tip (figure 8).

\subsection{Strategy of experimental-theoretical comparisons}

For this study, results coming from fourteen different experiments with Carbopol, and two different experiments with glucose, were aggregated (see table 1). These experiments were performed with different bottom velocities $u_{b}$ (in the range $25-150 \mathrm{~mm} . \mathrm{s}^{-1}$ ), different slope angles $\theta\left(11.9^{\circ}\right.$ and $\left.15.3^{\circ}\right)$, and different batches of Carbopol and glucose syrup. The yield stress $\tau_{c}$ of Carbopol samples varied between 5 and $12 \mathrm{~Pa}$. Accordingly, values of $B i$ and $R e$ achieved in the viscoplastic experiments spanned the ranges $0.4-1.1$ and $0.04-1.6$, respectively. Note that, in practice, numbers $B i$ and $R e$ tend to be inversely correlated in the experiments, such that high (resp. low) values of $B i$ are generally associated to low (resp. high) values of $R e$ (table 1). For the Newtonian experiments, the viscosity of glucose samples varied between 5 and 8 Pa.s, leading to values of $R e$ in the range $0.4-1.4$. Overall, the values of $\epsilon R e / F r^{2}$ are comprised between 0.1 and 


\begin{tabular}{rccccccccccc}
\multicolumn{2}{l}{ Carbopol } \\
exp. \# & $\tau_{c}$ & $K$ & $n$ & $\theta$ & $u_{b}$ & $H_{N}$ & $B i$ & $R e$ & $F r$ & $B i / \lambda$ & $\epsilon R / F r^{2}$ \\
HB1 & 13.3 & 9.8 & 0.40 & 15.3 & 22.6 & 14.6 & 1.13 & 0.044 & 0.061 & 0.35 & 0.17 \\
HB2 & 10.7 & 10.5 & 0.42 & 11.9 & 26.0 & 17.0 & 0.95 & 0.060 & 0.064 & 0.31 & 0.25 \\
HB3 & 13.3 & 9.8 & 0.40 & 15.3 & 37.0 & 16.2 & 0.95 & 0.098 & 0.095 & 0.32 & 0.18 \\
HB4 & 13.3 & 9.8 & 0.40 & 15.3 & 53.0 & 17.5 & 0.84 & 0.18 & 0.13 & 0.29 & 0.18 \\
HB5 & 9.0 & 6.9 & 0.42 & 15.3 & 46.0 & 12.4 & 0.80 & 0.19 & 0.13 & 0.28 & 0.13 \\
HB6 & 9.0 & 6.9 & 0.42 & 15.3 & 64.0 & 13.6 & 0.70 & 0.32 & 0.18 & 0.26 & 0.14 \\
HB7 & 13.3 & 9.8 & 0.40 & 15.3 & 78.0 & 19.0 & 0.74 & 0.34 & 0.18 & 0.27 & 0.19 \\
HB8 & 8.0 & 6.1 & 0.41 & 11.9 & 73.0 & 16.1 & 0.65 & 0.44 & 0.19 & 0.25 & 0.20 \\
HB9 & 13.3 & 9.8 & 0.40 & 15.3 & 128 & 21.6 & 0.62 & 0.76 & 0.28 & 0.24 & 0.21 \\
HB10 & 10.7 & 10.5 & 0.42 & 11.9 & 153 & 26.4 & 0.50 & 1.09 & 0.30 & 0.20 & 0.31 \\
HB11 & 9.0 & 6.9 & 0.42 & 15.3 & 123 & 15.9 & 0.56 & 0.95 & 0.32 & 0.22 & 0.15 \\
HB12 & 5.6 & 5.7 & 0.41 & 11.9 & 151 & 16.2 & 0.40 & 1.64 & 0.38 & 0.17 & 0.18 \\
HB13 & 8.0 & 6.0 & 0.43 & 15.3 & 148 & 15.5 & 0.50 & 1.37 & 0.39 & 0.20 & 0.14 \\
HB14 & 9.0 & 6.9 & 0.42 & 15.3 & 171 & 17.2 & 0.51 & 1.64 & 0.42 & 0.20 & 0.16
\end{tabular}

\begin{tabular}{rcccccccc}
\multicolumn{2}{l}{ Glucose syrup } \\
exp. \# & $\rho$ & $\eta$ & $\theta$ & $u_{b}$ & $H_{N}$ & $R e$ & $F r$ & $\epsilon R e / F r^{2}$ \\
Nt1 & 1383 & 5.6 & 15.3 & 75 & 18.6 & 0.35 & 0.18 & 0.20 \\
Nt2 & 1380 & 4.9 & 11.9 & 164 & 28.1 & 1.42 & 0.32 & 0.40
\end{tabular}

TABLE 1. Characteristics of the experiments performed with Carbopol and glucose syrup: fluid density $\rho\left(\mathrm{kg} \cdot \mathrm{m}^{-3}\right.$ : only indicated for glucose, taken at $1000 \mathrm{~kg} \cdot \mathrm{m}^{-3}$ for Carbopol), yield stress $\tau_{c}\left(\mathrm{~Pa}\right.$; for Carbopol), consistency $K$ (Pa.s ${ }^{n}$; for Carbopol), power-law index $n$ (for Carbopol), viscosity $\eta$ (Pa.s; for glucose), slope angle $\left(^{\circ}\right)$, bottom belt velocity $u_{b}\left(\mathrm{~mm} . \mathrm{s}^{-1}\right)$, steady uniform height $H_{N}(\mathrm{~mm})$, Bingham number $B i$ (for Carbopol), Reynolds number Re, Froude number $\mathrm{Fr}$, plug thickness in uniform regions $B i / \lambda$ (for Carbopol), and number $\epsilon \operatorname{Re} / \mathrm{Fr}^{2}$.

0.4. Although somewhat smaller than 1 , these values are systematically larger than $\epsilon$ (calculated for a typical length of $1 \mathrm{~m}$ ) by at least one order of magnitude, indicating that the experiments effectively comply with the flow regime considered in this study (see $\S 2.2$ ). Lastly, for reference, values of $F r$ and $B i / \lambda$ (plug thickness in uniform regions) are also indicated in table 1.

In the reference frame attached to channel bottom, the stationary surges generated in our setup correspond to travelling waves propagating at belt velocity $u_{b}$. Direct comparisons with theoretical predictions of models $M_{1}$ and $M_{1+\epsilon}$ obtained in $\S 3$, can thus be performed. To that aim, experimental measurements of longitudinal velocity $u$ are shifted by the value of $u_{b}$. Due to an experimental artefact, the depth-averaged velocities $\bar{u}$ obtained by integration of the shifted velocity profiles may differ from the expected value $u_{b}$ by a few percents (Freydier et al. 2017). To normalise experimental data consistently with theoretical predictions, measured velocities are thus rescaled by the actual, experimental values of $\bar{u}$ (constant along the surges). Similarly, positions $x_{f}$ and $y$ are rescaled by the value of uniform height $H_{N}$ directly measured on the surges, far from the tip.

Due to the nonphysical kink at the fake yield surface in the theoretical velocity profiles at order $O(\epsilon)$ (see $\S 3.2$ ), detailed comparisons with the experimental velocity profiles were not attempted in the viscoplastic case. Comparisons presented hereafter shall thus concentrate on (i) surface velocity $u_{s}$, and (ii) vertically-averaged shear rate in the pseudoplug layer $|\dot{\gamma}|_{p l}$. Note that we expect the evolution of $|\dot{\gamma}|_{p l}$ along the surge to be essentially unaffected by the singularity at $y=h-h_{p}$, since this quantity only depends on the longitudinal derivative $\partial_{x} u_{p l}^{(0)}$ [see (3.6)]. 


\subsection{Free-surface velocity}

Figure 9 shows comparisons between experimental surface velocities and predictions of models $M_{1}$ and $M_{1+\epsilon}$. Note that raw $u_{s}$-measurements generally present a slight systematic discrepancy with theoretical predictions far from the tip (see insets). This discrepancy, comprised within experimental errors, is attributed to uncertainties on the rheological parameters and, thus, on experimental $B i$ values (see figure 4, in which the dependence of $u_{s}$-predictions upon $B i$ is clearly observed). To better compare the modelled and experimental trends in the vicinity of the tip, datapoints in the main panel of figure 9 have been slightly shifted, so as to match theoretical predictions in the uniform zone.

As explained in $\S 3$, differences between predictions of models $M_{1}$ and $M_{1+\epsilon}$ get more pronounced when Bingham number $B i$ increases. Hence, let us first focus on an experiment characterised by a relatively large value of $B i$ and a small value of $R e$ (figure 9a). In this case, it is clearly observed that experimental values of $u_{s}$ increase faster than predictions of model $M_{1}$ when approaching the tip, with discrepancies becoming apparent for $x_{f}<2$ typically. On the contrary, experimental measurements are in good agreement with model $M_{1+\epsilon}$, even for the lowest values of $x_{f}$ at which measurements are available. The magnitudes of the $O(\epsilon)$ normal-stress and inertia correction terms are also represented in the figure. As expected, for this experiment, the $O(\epsilon)$ correction is dominated by the normal-stress term, while the inertia term remains much smaller and close to zero.

The better agreement provided by model $M_{1+\epsilon}$ close to the tip is also visible, though to a lesser extent, in figure $9 \mathrm{~b}$, which corresponds to an experiment with a lower value of $B i$ and a larger value of Re. Here, the magnitude of the inertia correction term is larger than in figure $9 \mathrm{a}$, such that the net difference between models $M_{1}$ and $M_{1+\epsilon}$ is less pronounced. Experimental data could be viewed as agreeing with either one of the two models, although the predictions of model $M_{1+\epsilon}$ again appear to better capture the increasing trend close to the tip.

Finally, figure $9 \mathrm{c}$ shows the case of a large Re number, for which the inertia and normalstress correction terms are almost of the same order. Here, predictions of the $M_{1}$ and $M_{1+\epsilon}$ models are essentially indistinguishable. Experimental data, however, deviate from these predictions close to the tip. Note that measurements seem to follow the evolution of the normal-stress correction term, suggesting that the inertia correction might be overestimated in this case.

To get a synthetic view of all experiments performed, the following error ratio was computed:

$$
r_{E}=\frac{\left|u_{s}^{\exp }-u_{s}^{M_{1+\epsilon}}\right|}{\left|u_{s}^{\exp }-u_{s}^{M_{1}}\right|},
$$

where $u_{s}^{\text {exp }}$ denotes the experimental surface velocity, and $u_{s}^{M_{1}}$ and $u_{s}^{M_{1+\epsilon}}$ denote the surface velocities predicted by models $M_{1}$ and $M_{1+\epsilon}$, respectively. Figure 10 shows values of $r_{E}$ obtained at $x_{f}=0.7$ as a function of $B i$ and $R e$ numbers. Similar trends are obtained for other values of $x_{f}$, as long as positions sufficiently close to the tip are considered. First, it is observed that $r_{E}$ displays values lower than 1, indicating that the match provided by model $M_{1+\epsilon}$ is systematically better than that obtained with model $M_{1}$. Moreover, the larger the values of $B i$ (or, correlatively, the lower the values of $R e$ ), the closer to 0 the values of $r_{E}$. This reflects an excellent agreement between experimental data and predictions of model $M_{1+\epsilon}$ for large $B i$ values. On the contrary, for low values of $B i$ (or large values of $R e$ ), the ratio $r_{E}$ remains close to 1 , meaning that 

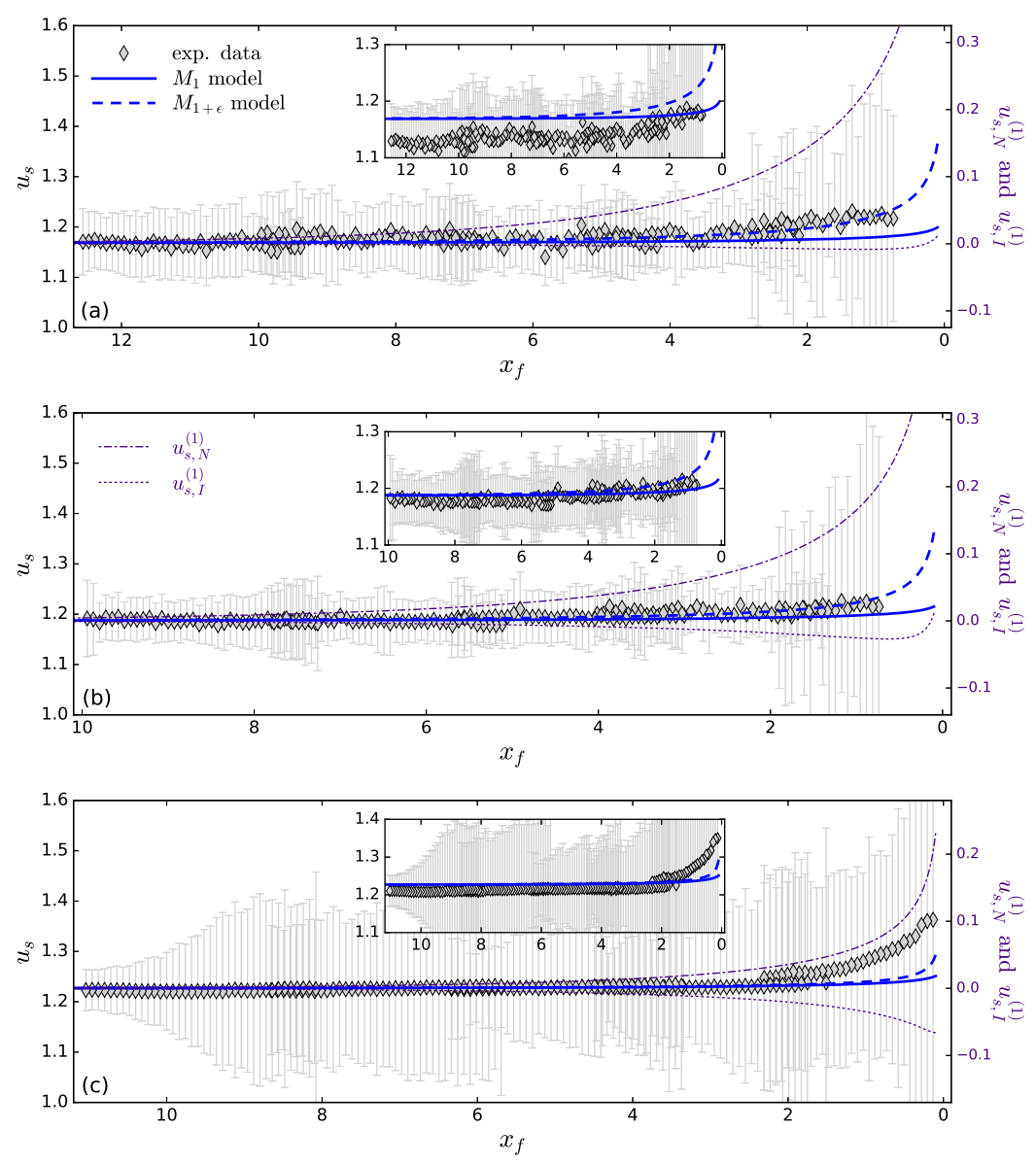

FIgURE 9. Free-surface velocity $u_{s}$ as a function of distance from the tip $x_{f}$ : comparison between experimental data and predictions of models $M_{1}$ and $M_{1+\epsilon}$. Error bars correspond to velocity measurement uncertainties. Light blue dotted and dot-dashed lines show contributions of the $O(\epsilon)$ normal-stress and inertia correction terms $u_{s, N}^{(1)}$ and $u_{s, I}^{(1)}$, respectively (scale on right axis). Insets display actual experimental data, while main panels show data corrected by a slight vertical shift (see text). (a) $B i=1.1, R e=0.04$ (exp. HB1). (b) $B i=0.8$ and $R e=0.2$ (exp. HB4). (c) $B i=0.5$ and $R e=1.4$ (exp. HB13).

the improvement brought by the $O(\epsilon)$ correction terms is relatively marginal in this case. In this limit of low $B i$ values, experimental data show significant discrepancies with both $M_{1}$ and $M_{1+\epsilon}$ models.

\subsection{Pseudo-plug shear rate}

Figure 11 shows examples of shear rate $|\dot{\gamma}|$ computed by direct differentiation of the measured velocity fields (accounting for vertical and longitudinal derivative terms). To improve clarity, values of $|\dot{\gamma}|$ are normalised by the typical level of experimental noise $\dot{\gamma}_{c}$ (variable among the experiments), and the colour scale is thresholded for $|\dot{\gamma}| / \dot{\gamma}_{c}<1$. Hence, dark-blue/black areas correspond to the apparent unsheared layer that can be determined from the experiments. Far from the tip, this apparent unsheared layer is thicker than the predicted pseudo-plug, due to the thresholding at $\dot{\gamma}_{c}$. In the tip region, on the contrary, it is observed that the experimental unsheared layer thins much faster 

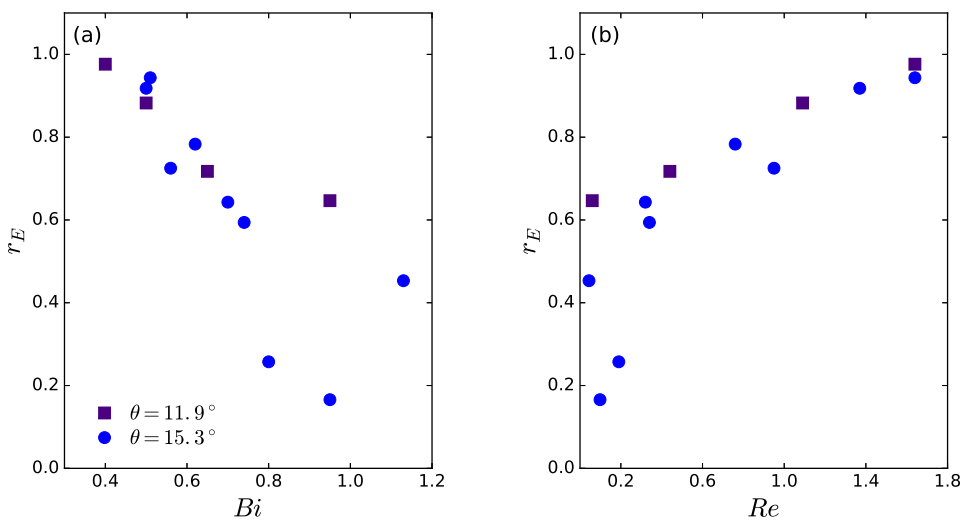

FiguRE 10. Error ratio $r_{E}$ (see text) computed at $x_{f}=0.7$ as a function of (a) Bingham number $B i$ and (b) Reynolds number Re, for the 14 experiments performed with Carbopol (see table 1).
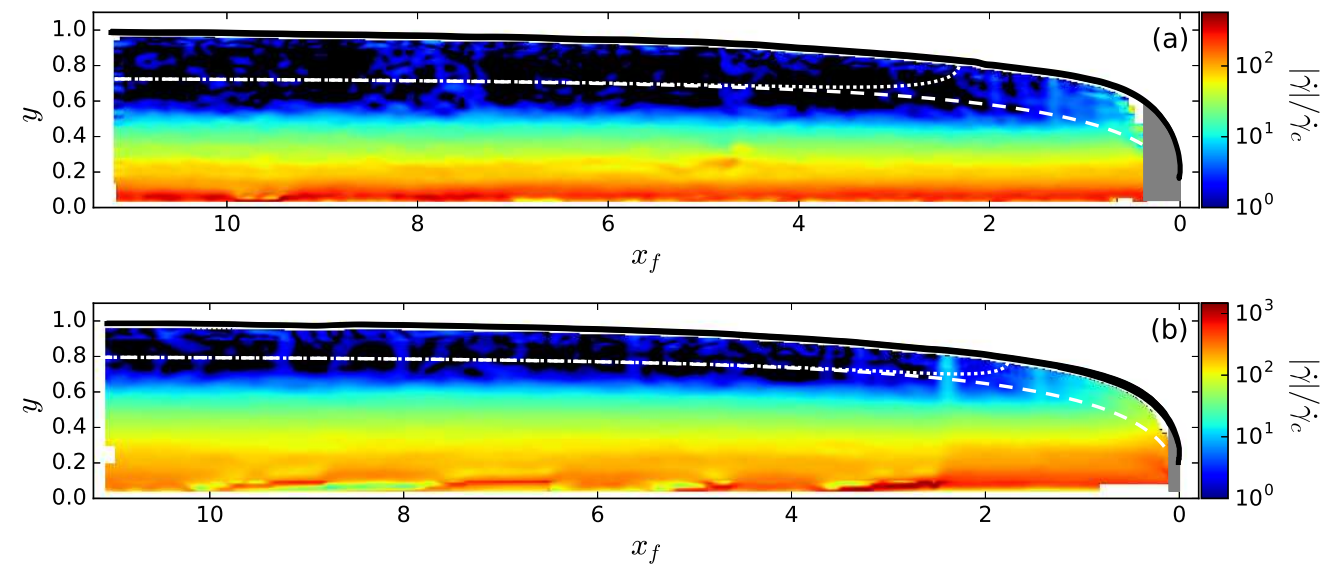

FiguRE 11. Experimental shear rate $|\dot{\gamma}|$ normalised by typical measurement noise level $\dot{\gamma}_{c}$. The white dashed line represents the fake yield surface predicted by model $M_{1+\epsilon}$ (essentially indistinguishable from that predicted by model $M_{1}$ ), while the white dotted line represents the predicted iso-contour $|\dot{\gamma}|=\dot{\gamma}_{c}$ in the pseudo-plug (see $\S 3.3$ ). (a) $B i=0.7, R e=0.3$ (exp. HB7, $\left.\dot{\gamma}_{c}=0.033\right)$. (b) $B i=0.5, R e=1.4$ (exp. HB13, $\left.\dot{\gamma}_{c}=0.013\right)$.

than the pseudo-plug, and disappears for $x_{f}<1-2$ typically. Interestingly, this feature appears to be consistent with the abrupt collapse of the theoretical effective unsheared layer obtained from model $M_{1+\epsilon}$ (see $\S 3.3$ ). If this theoretical effective unsheared layer is defined on the base of the iso-value of shear rate corresponding to measurement noise $\dot{\gamma}_{c}$, both the overall shape of the layer and the location of the collapse appear in reasonable agreement with experimental data (figure 11).

More quantitatively, figure 12 compares the evolution of the vertically-averaged shear rate $|\dot{\gamma}|_{p l}$ measured in the pseudo-plug with predictions of model $M_{1+\epsilon}$. As for their theoretical counterparts (see $\S 3.3$ ), experimental values of $|\dot{\gamma}|_{p l}$ are computed by averaging all $|\dot{\gamma}|$-measurements obtained within the boundaries of the theoretical pseudo-plug for a given position $x_{f}$. In agreement with the model, a clear increase of $|\dot{\gamma}|_{p l}$ in the vicinity of the tip is visible in the data. Measurements appear to be nicely captured by the theoretical predictions for small to moderate values of $R e$ (figure $12 \mathrm{a}$ and $12 \mathrm{~b}$ ). Note that the slightly less compelling agreement observed for the largest value of $B i$ (figure 12a), 

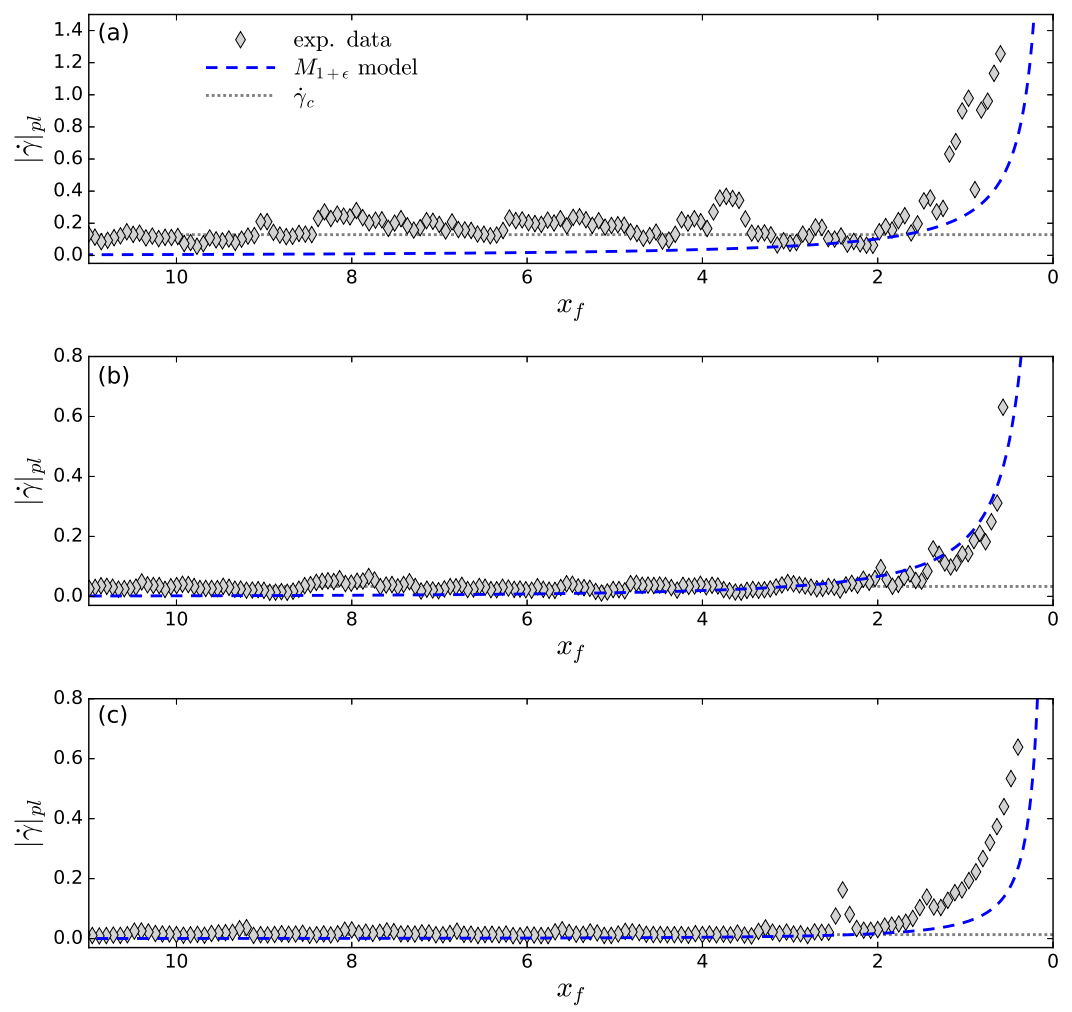

FiguRE 12. Vertically-averaged shear rate in the pseudo-plug $|\dot{\gamma}|_{p l}$ as a function of distance from the tip $x_{f}$ : comparison between experimental data and predictions of model $M_{1+\epsilon}$. The grey dotted line indicates the typical level of measurement noise $\dot{\gamma}_{c}$. (a) $B i=0.95, R e=0.06$ (exp. HB2). (b) $B i=0.7, R e=0.3$ (exp. HB7). (c) $B i=0.5, R e=1.4$ (exp. HB13).

can be explained by a larger noise level for this experiment. Conversely, as for surface velocities, predictions of model $M_{1+\epsilon}$ appear to underestimate the data for large values of $R e$ (figure 12c).

\section{Discussion}

To the best of our knowledge, this paper presents the first complete asymptotic expansion, up to order $O(\epsilon)$, of longitudinal velocity $u$ and flow discharge $q$ in a thin free-surface viscoplastic surge. Our analysis expands on the previous work of FernandezNieto et al. (2010) in two respects: (i) the general case of a Herschel-Bulkley constitutive law (with $n>0$ ) is treated, and (ii) we consider a flow regime in which $\epsilon R e / F r^{2}=$ $O(1)$, whereas this term was regarded as $O(\epsilon)$ by Fernandez-Nieto et al. (2010). This latter assumption provides more flexibility to the leading-order solution, which coincides with a lubrication approximation characterised by a variable pseudo-plug thickness $h_{p}$ depending on $\partial_{x} h$. Such a feature is required to model, e.g., surge fronts, in which pseudo-plug thickness cannot be regarded as constant. This flow regime also matches that typically achieved in the experiments used for comparisons in $\S 4$. As a counterpart, the assumption $\epsilon R e / \mathrm{Fr}^{2}=O(1)$ leads to the appearance of factors proportional to $\partial_{x} h_{p}$ and $\partial_{t} h_{p}$ in the $O(\epsilon)$ correction terms. It is straightforward to check that the the expressions of correction terms derived in $\S 2$, effectively reduce to those obtained by Fernandez-Nieto 
et al. (2010) when setting $\epsilon R e / F r^{2}=0$ (and thus replacing $\Lambda$ by $\lambda$ and cancelling out all terms in $\left.\partial_{x, t} \Lambda\right)$.

\subsection{Influence of $O(\epsilon)$ correction terms}

Two sets of $O(\epsilon)$ correction terms, with different physical origins, affect the asymptotic expansions of $u$ and $q$. Correction terms related to inertia effects also arise in the case of Newtonian or power-law fluids (Fernandez-Nieto et al. 2010; Noble \& Vila 2013; Boutounet et al. 2016). Normal-stress correction terms, on the contrary, are specific to the viscoplastic character of the fluid, since they are directly related to the existence of the pseudo-plug layer. Note that in the flow regime considered here, and unlike in the work of Fernandez-Nieto et al. (2010), these normal-stress corrections affect the velocity profile in both the pseudo-plug and the sheared layer underneath.

The relative influence of these $O(\epsilon)$ correction terms was investigated in the specific case of travelling-wave solutions, focusing on the tip region. The free-surface shape was shown to be only weakly sensitive to $O(\epsilon)$ corrections, regardless of the considered $B i$ and $R e$ values (see figure 2). Interestingly, on the contrary, a significant effect of both inertia and normal-stress correction terms was observed on the velocity profiles, all the more for small values of $n$. This markedly contrasts with the case of Newtonian fluids (see Appendix B) or power-law fluids (not shown), for which leading-order and $O(\epsilon)$ velocity profiles remain essentially indiscernible, even close to the tip. In fact, an increase of Bingham number $B i$ was shown to result in an increased influence of both inertia and normal-stress correction terms (see figure 3). Even though they arise from different physical origins, these correction terms are strongly coupled, and both increase with the viscoplastic character of the fluid. It was also observed that the two correction terms generally act in opposite directions, such that their effects tend to counterbalance for sufficiently large values of $B i$ and $R e$. As a consequence, the net difference between predictions of leading-order and $O(\epsilon)$ models, varies in a complex and non-monotonic manner as a function of $B i$ and $R e$.

A shortcoming of the present study lies in the non-differentiability across the fake yield surface of the velocity profiles predicted at $O(\epsilon)$ (see figure 3). This singularity arises from the normal-stress correction terms in the pseudo-plug, more precisely from the factor $\left[1-\left((h-y) / h_{p}\right)^{2}\right]^{1 / 2}$ in $(2.34)$. Note that this factor is independent of $n$, such that the same singularity is also present in past studies considering Bingham fluids (Balmforth \& Craster 1999; Fernandez-Nieto et al. 2010). As discussed in these previous studies, construction of more regular velocity profiles would require a proper asymptotic matching, through the introduction of a transition layer, between the sheared layer and the pseudo-plug. The complete formalisation of this asymptotic matching for general values of $n$, appears a promising prospect to further extend the results presented in this paper, and shall constitute the subject of future work.

\subsection{Agreement with experimental data}

Regardless of the number of terms retained in the asymptotics, the validity of the thin-layer assumption is clearly expected to break down in the vicinity of surge tip, where $\left|\partial_{x} h\right| \rightarrow \infty$. Note that, in absence of additional regularising conditions, even the primitive Cauchy equations become ill-posed at moving contact lines (e.g., Dussan V 1976). Nevertheless, since $\left|\partial_{x} h\right|$ typically remains less than 1 for $x_{f}>0.1$, well-ordered asymptotic expansions, and meaningful comparisons with experimental data, could be obtained relatively close to the tip. It ought to be mentioned here that, as shown in figure 13, a slight but systematic discrepancy is observed between measured and 


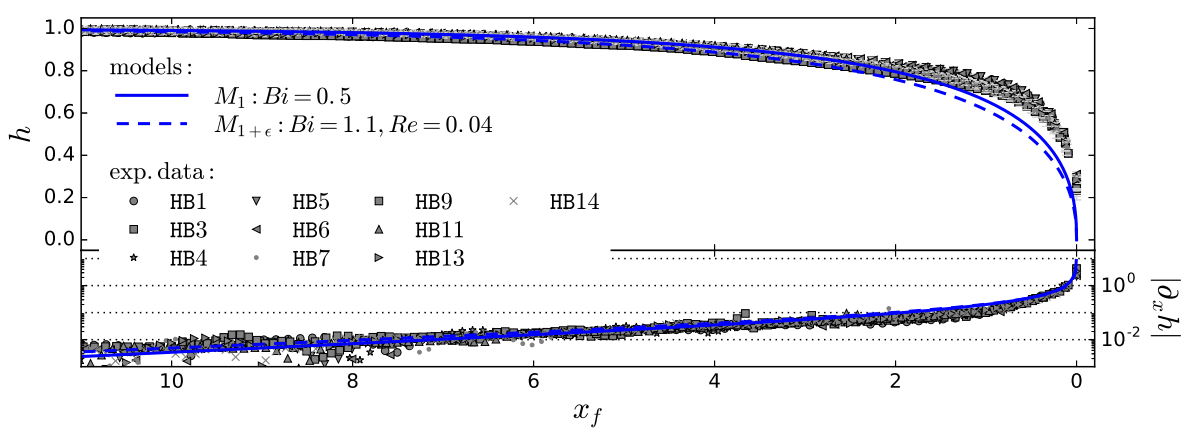

FiguRE 13. Evolution of flow height $h$ and free-surface slope $\partial_{x} h$ as a function of distance from the tip $x_{f}$ : comparison between all experimental data obtained for $\theta=15.3^{\circ}$ (see table 1 ) and predictions of models $M_{1}$ and $M_{1+\epsilon}$. For the sake of clarity, only the two model predictions that differ most from one another, are shown.

theoretical free-surface shapes for $x_{f}<2$, i.e. when $\left|\partial_{x} h\right|>0.1$, typically (see also Freydier et al. 2017). Arguably, this discrepancy could be interpreted as the signature of an early break-down of the thin-layer assumption. Let us however note that, in the Newtonian case, a much better agreement is recovered between measured and predicted free-surface shapes, even in the zone where $\left|\partial_{x} h\right|>0.1$ (see Appendix B). As discussed in Freydier et al. (2017), this discrepancy on free-surface shapes observed with Carbopol, might also be due to more complex rheological trends of the material that are not accounted for by Herschel-Bulkley law.

Theoretical velocity profiles used for comparisons with experimental data were constructed from the modelled free-surface shapes. Using instead measured values of $h$, would have resulted in predicted velocity profiles characterised by non-constant depthaverages $\bar{u}$ in the tip region, and thus more difficult to compare with experimental data. As already reported by Freydier et al. (2017), for viscoplastic fluids, the leading-order approximation appears to be in excellent agreement with longitudinal velocity measurements for $x_{f}>1-2$, typically. Closer to the tip, clear discrepancies are visible, notably a rapid collapse of the apparent unsheared layer identified in the experiments. In contrast, in the Newtonian case, the agreement between experimental velocity measurements and the leading-order approximation remains excellent all along the surge, including close to the tip (Appendix B). Note that similar comparisons between lubrication models and experiments were attempted by Andreini et al. (2012), although with less clear conclusions due to somewhat noisier data regarding the evolution of the unsheared zone in the tip, and strong discrepancies in average velocity.

The main result of the present study is the clearly improved match with experimental data obtained, for viscoplastic fluids, when $O(\epsilon)$ correction terms are included in the expansion. Importantly, the $O(\epsilon)$ approximation captures the abrupt collapse of the unsheared layer in the tip (see figure 11). The increase in shear rate and surface velocity are also quantitatively reproduced by the $O(\epsilon)$ model, and this down to values of $x_{f}$ as small as $0.1-0.2$ (see figures 12 and 9 ). Note that this good agreement regarding velocity and shear-rate data, tends to confirm that discrepancies observed on free-surface shapes for $x_{f}<1-2$ (figure 13) are not imputable to a break-down of the asymptotics. The $O(\epsilon)$ model shows nevertheless a tendency to underestimate both velocity and shearrate measurements for $R e>1-1.5$, typically, as if the counterbalancing effect between inertia and normal-stress correction terms was overly pronounced in this case. Overall, the normal-stress correction appears to play a critical role in the improvement brought by 
the $O(\epsilon)$ approximation. Conversely, we suspect the magnitude of the inertia correction to be overestimated in our approach. It is however likely that the predictive capabilities of the $O(\epsilon)$ approximation would also be further improved with a more accurate free-surface model.

\section{Conclusions}

This paper presents consistent asymptotic expansions, up to $O(\epsilon)$ in flow aspect ratio $\epsilon$, of the velocity field in free-surface surges of viscoplastic fluids. At leading-order, the expansion coincides with a lubrication approximation. The influence of $O(\epsilon)$ correction terms was investigated in the case of travelling-wave solutions, and detailed comparisons with the unique, high-resolution, experimental dataset of Freydier et al. (2017) were drawn. The main conclusions of the study can be summarised as follows:

(i) While leading-order and $O(\epsilon)$ approximations are essentially indistinguishable for Newtonian and power-law fluids, the influence of $O(\epsilon)$ correction terms is enhanced by the viscoplastic character of the fluid, notably for small values of power index $n$. Viscoplastic flows thus provide interesting case studies, in which the differences between leading-order and $O(\epsilon)$ asymptotic models are potentially amenable to experimental measurements.

(ii) For viscoplastic fluids, inclusion of the $O(\epsilon)$ correction terms in the asymptotic analysis, qualitatively alters the description of the flow in the tip region. While the leading-order model predicts the existence of an unsheared pseudo-plug zone all along the surge, $O(\epsilon)$ correction terms entail a significant increase of the predicted shear rate, and an effective elimination of the unsheared zone, in the tip region.

(iii) For viscoplastic fluids, remarkable agreement is observed between the $O(\epsilon)$ model and experimental measurements of surface velocity and shear rate, and this even in close vicinity of surge tip, down to $x_{f} / H_{N} \approx 0.1$. On the contrary, the leading-order model fails to reproduce evolution of surface velocity and shear rate for $x_{f} / H_{N}<1-2$, typically. For Newtonian fluids, both leading-order and $O(\epsilon)$ models nicely capture experimental data for all $x_{f} / H_{N}>0.1$.

(iv) A more regular matching between the sheared layer and the pseudo-plug would be needed to construct differentiable velocity profiles above leading order in the viscoplastic case. This improvement shall allow us to achieve direct comparisons with measured velocity profiles.

To conclude, let us recall that the obtained $O(\epsilon)$ expansions of longitudinal velocity $u$ and discharge $q$, constitute pre-requisites for the formulation of consistent closure terms in shallow-water models (see Introduction). Beyond the argument of mathematical consistency, the results obtained in this study also suggest that, for viscoplastic fluids, inclusion of $O(\epsilon)$ terms might have a noticeable impact on the predictions of these models in terms of, e.g., velocity values. Proper derivation of consistent shallow-water models for viscoplastic fluids, and comparisons thereof with experimental data, shall be the subject of upcoming studies. Note however that the single-equation model $M_{1+\epsilon}$ obtained in $\S 3.1$, can already be regarded as a specialised version of a consistent shallow-water model adapted to the case of travelling-wave solutions (for which $q$ is enslaved to $h$ ). Let us also mention that alternative expressions of the $O(\epsilon)$ correction terms, which are all equivalent up to order $O(\epsilon)$, could be proposed. Accordingly, a whole family of shallow-water models can actually be constructed (see also Boutounet et al. 2016). In this work, differential identity (A 1) was used to retain only factors in $\partial_{x} h$ and $\partial_{x, t} \Lambda$ in the final expressions of velocity profiles at $O(\epsilon)$. The exact influence of these algebraic manipulations on the final predictions of shallow-water models, remains to be properly investigated. 


\section{Acknowledgements}

Financial support from AGIR PEPS project MIGRAIN (Univ. Grenoble Alpes) and CNRS (InFIniti program) is acknowledged. IRSTEA is member of Labex TEC21 (Investissements d'Avenir - Grant Agreement ANR-11-LABX-0030) and Labex Osug@2020 (Investissements d'Avenir - Grant Agreement ANR-10-LABX-0056).

Declaration of Interests. None.

\section{Appendix A. Expressions of $\mathcal{U}_{I}$ and $\mathcal{Q}_{I}$}

In this appendix, we provide explicit expressions for the inertia-related correction functions $\mathcal{U}_{I}$ and $\mathcal{Q}_{I}$ involved in the expansions of longitudinal velocity $u$ and flow discharge $q$ at $O(\epsilon)$ (see $\S 2.5$ ). As a preamble, let us note that a useful differential identity, valid at order $O(1)$, derives from the depth-integrated mass conservation equation (2.16). Inserting the leading-order approximation of discharge $q^{(0)}$ given by $(2.24)$, leads to:

$$
\begin{aligned}
& \partial_{t} h=-\Lambda^{1 / n}\left(h-h_{p}\right)^{(n+1) / n}\left(1+\varpi_{p}\right) \partial_{x} h \\
&-\Lambda^{(1-n) / n}\left(h-h_{p}\right)^{(2 n+1) / n}\left(\frac{1}{2 n+1}+\frac{2}{n+1} \varpi_{p}+\varpi_{p}^{2}\right) \partial_{x} \Lambda+\ldots,
\end{aligned}
$$

with $\varpi_{p}=h_{p} /\left(h-h_{p}\right)$. As in Fernandez-Nieto et al. (2010), this identity can be used to simplify the expressions of $\mathcal{U}_{I}$ and $\mathcal{Q}_{I}$, replacing factors proportional to $\partial_{t} h$ by factors proportional to $\partial_{x} h$ and $\partial_{x} \Lambda$.

The function $\mathcal{U}_{I}$ is obtained from (2.44) and the expressions of leading-order longitudinal and vertical velocities $u^{(0)}$ and $v^{(0)}$. The lengthy algebraic calculations were performed with wxMaxima symbolic software. The final expression writes:

$$
\begin{aligned}
\mathcal{U}_{I}(x, \zeta, t)=\Lambda^{(3-n) / n} & \left(h-h_{p}\right)^{(2 n+3) / n} q_{\mathcal{U}, 1}\left(\zeta, \varpi_{p}\right) \partial_{x} h \\
+ & \Lambda^{(3-2 n) / n}\left(h-h_{p}\right)^{(3 n+3) / n} q_{\mathcal{U}, 2}\left(\zeta, \varpi_{p}\right) \partial_{x} \Lambda \\
& \quad-\Lambda^{(2-2 n) / n}\left(h-h_{p}\right)^{(2 n+2) / n} q_{\mathcal{U}, 1}\left(\zeta, \varpi_{p}\right) \partial_{t} \Lambda,
\end{aligned}
$$

where $q_{\mathcal{U}, 1}\left(\zeta, \varpi_{p}\right)$ and $q_{\mathcal{U}, 2}\left(\zeta, \varpi_{p}\right)$ are polynomials in $\zeta$ and $\varpi_{p}$ :

$$
\begin{aligned}
q_{\mathcal{U}, 1}\left(\zeta, \varpi_{p}\right)= & \left(\frac{3 n+2}{2(n+1)^{2}(2 n+1)}-\frac{1}{(n+1)^{2}} \zeta^{(n+1) / n}+\frac{n}{2(n+1)^{2}(2 n+1)} \zeta^{(2 n+2) / n}\right) \\
& +\varpi_{p}\left(\frac{n+4}{(n+1)(n+2)}-\frac{1}{n+1} \zeta^{1 / n}-\frac{1}{n+1} \zeta^{(n+1) / n}+\frac{n}{(n+1)(n+2)} \zeta^{(n+2) / n}\right) \\
& +\varpi_{p}^{2}\left(1-\zeta^{1 / n}\right) \\
q_{\mathcal{U}, 2}\left(\zeta, \varpi_{p}\right)= & \left(\frac{12+24 n+3 n^{2}-11 n^{3}}{6(n+1)^{3}(2 n+1)(3 n+2)}-\frac{1+n-n^{2}}{(n+1)^{3}(2 n+1)} \zeta^{(n+1) / n}+\frac{n(1-n)}{2(n+1)^{3}(2 n+1)} \zeta^{(2 n+2) / n}\right. \\
& \left.+\frac{n^{3}}{3(n+1)^{3}(2 n+1)(3 n+2)} \zeta^{(3 n+3) / n}\right) \\
+ & \varpi_{p}\left(\frac{12+18 n-7 n^{2}-2 n^{3}}{2(n+1)^{2}(n+2)(2 n+1)}-\frac{1+n-n^{2}}{(n+1)^{2}(2 n+1)} \zeta^{1 / n}-\frac{2-n}{(n+1)^{2}} \zeta^{(n+1) / n}\right. \\
& \left.+\frac{n(1-n)}{(n+1)^{2}(n+2)} \zeta^{(n+2) / n}+\frac{n}{2(n+1)^{2}(2 n+1)} \zeta^{(2 n+2) / n}\right) \\
+ & \varpi_{p}^{2}\left(\frac{6-n^{2}}{(n+1)(n+2)}-\frac{2-n}{n+1} \zeta^{1 / n}-\frac{1}{n+1} \zeta^{(n+1) / n}+\frac{n}{(n+1)(n+2)} \zeta^{(n+2) / n}\right) \\
+ & \varpi_{p}^{3}\left(1-\zeta^{1 / n}\right) .
\end{aligned}
$$

It can be checked that $q_{\mathcal{U}, 1}\left(1, \varpi_{p}\right)=q_{\mathcal{U}, 2}\left(1, \varpi_{p}\right)=0$, as imposed by the no-slip basal boundary condition. 
The function $\mathcal{Q}_{I}$ is calculated by a further integration of $\mathcal{U}_{I}: \mathcal{Q}_{I}=\left(h-h_{p}\right) \int_{0}^{1} \mathcal{U}_{I}(\zeta) d \zeta+$ $h_{p} \mathcal{U}_{I}(0)$. The final expression writes:

$$
\begin{aligned}
\mathcal{Q}_{I}(x, t)=\Lambda^{(3-n) / n} & \left(h-h_{p}\right)^{(3 n+3) / n} q_{\mathcal{Q}, 1}\left(\varpi_{p}\right) \partial_{x} h \\
& +\Lambda^{(3-2 n) / n}\left(h-h_{p}\right)^{(4 n+3) / n} q_{\mathcal{Q}, 2}\left(\varpi_{p}\right) \partial_{x} \Lambda \\
& -\Lambda^{(2-2 n) / n}\left(h-h_{p}\right)^{(3 n+2) / n} q_{\mathcal{Q}, 1}\left(\varpi_{p}\right) \partial_{t} \Lambda
\end{aligned}
$$

with

$$
\begin{aligned}
q_{\mathcal{Q}, 1}\left(\varpi_{p}\right)= & \frac{2}{(2 n+1)(3 n+2)}+\frac{5 n+3}{(n+1)^{2}(2 n+1)} \varpi_{p}+\frac{2(n+3)}{(n+1)(n+2)} \varpi_{p}^{2}+\varpi_{p}^{3} \\
q_{\mathcal{Q}, 2}\left(\varpi_{p}\right)= & \frac{2\left(3+4 n-3 n^{2}\right)}{(2 n+1)^{2}(3 n+2)(4 n+3)}+\frac{24+57 n+18 n^{2}-22 n^{3}}{3(n+1)^{3}(2 n+1)(3 n+2)} \varpi_{p}+\frac{3\left(4+7 n-n^{2}-n^{3}\right)}{(n+1)^{2}(n+2)(2 n+1)} \varpi_{p}^{2} \\
& +\frac{8+n-n^{2}}{(n+1)(n+2)} \varpi_{p}^{3}+\varpi_{p}^{4} .
\end{aligned}
$$

\section{Appendix B. Newtonian case}

This appendix presents: (i) the specialised version of the asymptotic expansion derived in $\S 2$ for the case of Newtonian fluids, and (ii) comparisons with the experimental results obtained with glucose syrup (see table 1 ).

\section{B.1. Velocity profile and discharge}

Setting $B i=0$ (thus $h_{p}=0$ ) and $n=1$ in (2.22), a classical parabolic profile is recovered for longitudinal velocity $u$ at leading order:

$$
u^{(0)}=\frac{1}{2} \Lambda h^{2}\left(1-\zeta^{2}\right)
$$

with $\zeta=1-y / h$. Accordingly, leading-order discharge $q^{(0)}$ writes:

$$
q^{(0)}=\frac{1}{3} \Lambda h^{3} .
$$

At $O(\epsilon)$, only inertia correction terms are present, such that (2.46) reduces to:

$$
u^{(1)}=\operatorname{Re} \mathcal{U}_{I}(\zeta)
$$

with here:

$$
\begin{array}{r}
\mathcal{U}_{\mathcal{I}}(\zeta)=\Lambda^{2} h^{5}\left(\frac{5}{24}-\frac{1}{4} \zeta^{2}+\frac{1}{24} \zeta^{4}\right) \partial_{x} h+\Lambda h^{6}\left(\frac{7}{180}-\frac{1}{24} \zeta^{2}+\frac{1}{360} \zeta^{6}\right) \partial_{x} \Lambda \\
-h^{4}\left(\frac{5}{24}-\frac{1}{4} \zeta^{2}+\frac{1}{24} \zeta^{4}\right) \partial_{t} \Lambda .
\end{array}
$$

Similarly, the $O(\epsilon)$ correction on discharge writes:

$$
q^{(1)}=\epsilon \operatorname{Re} \mathcal{Q}_{I}
$$

with

$$
\mathcal{Q}_{I}=\frac{2}{15} \Lambda^{2} h^{6} \partial_{x} h+\frac{8}{315} \Lambda h^{7} \partial_{x} \Lambda-\frac{2}{15} h^{5} \partial_{t} \Lambda .
$$

\section{B.2. Travelling-wave solutions}

The same choice of characteristic scales as in $\S 3$, results here in the identity $\lambda=3$. Like for the viscoplastic case, two free-surface models can be constructed from the relation $q=h_{t w}$ characterising travelling-wave solutions. Model $M_{1}$ is based on the leading-order approximation (B 2) of $q$. This model writes here:

$$
M_{1}: \quad \cot \theta \frac{d h_{t w}}{d x_{f}}=\frac{1}{h_{t w}^{2}}-1,
$$




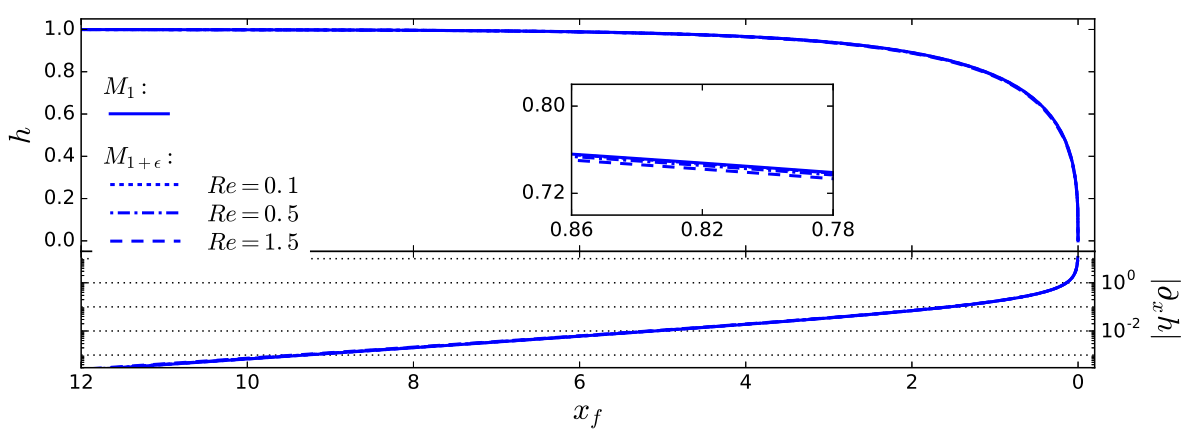

FIgURE 14. Free-surface shapes predicted by models $M_{1}$ and $M_{1+\epsilon}$ for a Newtonian fluid: evolution of flow height $h$ and derivative $\partial_{x} h$ versus distance from the tip $x_{f}$. Solutions of model $M_{1+\epsilon}$ computed for three different values of $R e$ are shown $\left(\theta=15^{\circ}\right)$. Inset shows a close-up on the minute differences among the solutions.

where we recall that $x_{f}=t-x$ with the considered choice of characteristic scales. With the boundary condition $h_{t w}(0)=0$, this equation admits an implicit analytical solution:

$$
x_{f}=\cot \theta\left[\frac{1}{2} \ln \left(\frac{1+h_{t w}}{1-h_{t w}}\right)-h_{t w}\right] .
$$

Hence, the normalised free surface predicted by model $M_{1}$ is completely independent of fluid rheological parameters in the Newtonian case. Model $M_{1+\epsilon}$ is based on the $O(\epsilon)$ approximation of discharge $q^{(0)}+q^{(1)}$, with $q^{(1)}$ given by (B 5). The corresponding secondorder ODE takes here the following form:

$$
M_{1+\epsilon}: \quad 1+\cot \theta \frac{d h_{t w}}{d x_{f}}+\operatorname{Re} \hat{\mathcal{Q}}_{I}-\frac{1}{h_{t w}^{2}}=0,
$$

with

$$
\hat{\mathcal{Q}}_{I}=\frac{\mathcal{Q}_{I}}{h_{t w}^{3}}=-\frac{2}{15} \Lambda_{t w}^{2} h_{t w}^{3} \frac{d h_{t w}}{d x_{f}}-\left(\frac{8}{315} \Lambda_{t w} h_{t w}^{4}+\frac{2}{15} h_{t w}^{2}\right) \cot \theta \frac{d^{2} h_{t w}}{d x_{f}^{2}}
$$

and $\Lambda_{t w}=3\left(1+\cot \theta d h_{t w} / d x_{f}\right)$.

\section{B.3. Model predictions}

Figure 14 shows that free surfaces computed with models $M_{1}$ and $M_{1+\epsilon}$ are essentially indistinguishable in the Newtonian case, and this regardless of the value of $R e$ (in the range $0-1.5)$. Concerning free-surface velocity $u_{s}$, a constant value of $u_{s}$ is predicted by model $M_{1}$, while values of $u_{s}$ computed with model $M_{1+\epsilon}$ display a decreasing trend towards the tip (figure 15). Note however that the differences between the two models remain typically tenfold smaller than in the viscoplastic case (for similar values of Re: see $\S 3.2)$. As in figure $4, u_{s}$-curves in figure 15 are only represented for $x_{f}>0.1$, i.e. in the domain where $\left|\partial_{x} h\right|$ remains typically less than 1 (see figure 14).

\section{B.4. Comparison with experimental data}

Characteristics of the experiments performed with Newtonian samples (glucose syrup) are summarised in table 1. As shown in figure 16, experimentally-measured front shapes are in good agreement with the theoretical predictions provided by either model $M_{1}$ or $M_{1+\epsilon}$. The two models tend here to slightly overestimate the data, but the differences remain significantly smaller than in the viscoplastic case (compare with figure 13). 


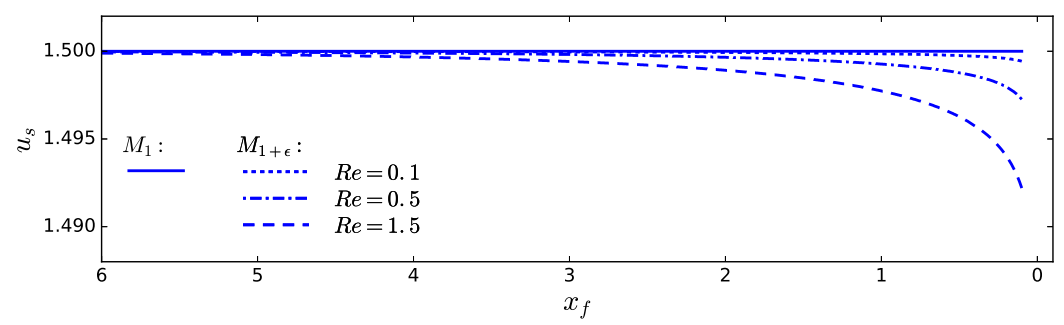

FiguRE 15. Evolution of free-surface velocity $u_{s}$ predicted by models $M_{1}$ and $M_{1+\epsilon}$ as a function of distance from the tip $x_{f}$ for a Newtonian fluid. Solutions of model $M_{1+\epsilon}$ computed for three different values of $R e$ are shown $\left(\theta=15^{\circ}\right)$.

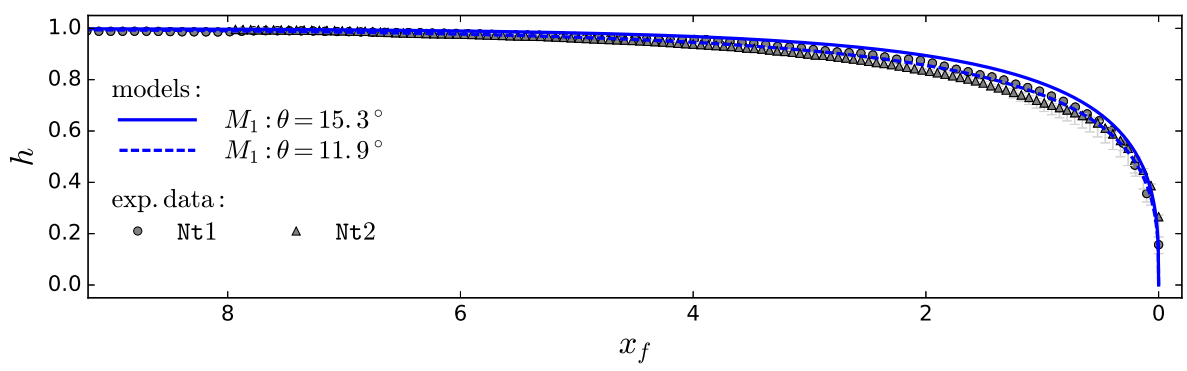

FIGURE 16. Free-surface shape in the Newtonian case: comparison between experimental data (see table 1) and theoretical predictions. As predictions of models $M_{1}$ and $M_{1+\epsilon}$ are indistinguishable, only the former are represented for the two values of slope angle $\theta$.

Similarly, as shown in figure 17, measured velocity profiles are remarkably captured by both asymptotic models. At the scale used in the figure, the velocity profiles predicted by models $M_{1}$ and $M_{1+\epsilon}$ are indistinguishable. The agreement with experimental data remains excellent even for the smallest values of $x_{f}$ investigated (figures $17 \mathrm{c}$ and $17 \mathrm{e}$ ).

\section{REFERENCES}

Amaouche, M., Duema, A. \& Ait Aberrahmane, H. 2012 Film flow for power-law fluids: modeling and linear stability. Eur. J. Mech. (B/Fluids) 34, 70-84.

Ancey, C. 2007 Plasticity and geophysical flows: A review. J. Non-Newton. Fluid 142, 4-35.

Ancey, C., Andreini, N. \& Epely-Chauvin, G. 2012 Viscoplastic dambreak waves: Review of simple computational approaches and comparison with experiments. Adv. Water Res. 48, 79-91.

Ancey, C. \& Cochard, S. 2009 The dam-break problem for Herschel-Bulkley viscoplastic fluids down steep flumes. J. Non-Newton. Fluid 158, 18-35.

Ancey, C., Cochard, S. \& Andreini, N. 2009 The dam-break problem for viscous fluids in the high-capillary-number limit. J. Fluid Mech. 624, 1-22.

Ancey, C., Cochard, S., Rentschler, M. \& Wiederseiner, S. 2007 Existence and features of similarity solutions for non-Boussinesq gravity currents. Physica D 226, 32-54.

Andreini, N., Epely-Chauvin, G. \& Ancey, C. 2012 Internal dynamics of Newtonian and viscoplastic fluid avalanches down a sloping bed. Phys. Fluids 24, 053101.

Balmforth, N. J. \& Craster, R. V. 1999 A consistent thin-layer theory for Bingham plastics. J. Non-Newton. Fluid 84, 65-81.

Balmforth, N. J., Craster, R. V., Rust, A. C. \& Sassi, R. 2006 Viscoplastic flow over an inclined surface. J. Non-Newton. Fluid 139, 103-127.

Balmforth, N. J., FrigaArd, I. A. \& Ovarlez, G. 2014 Yielding to stress: Recent developments in viscoplastic fluid mechanics. Annu. Rev. Fluid Mech. 46, 121-146. 

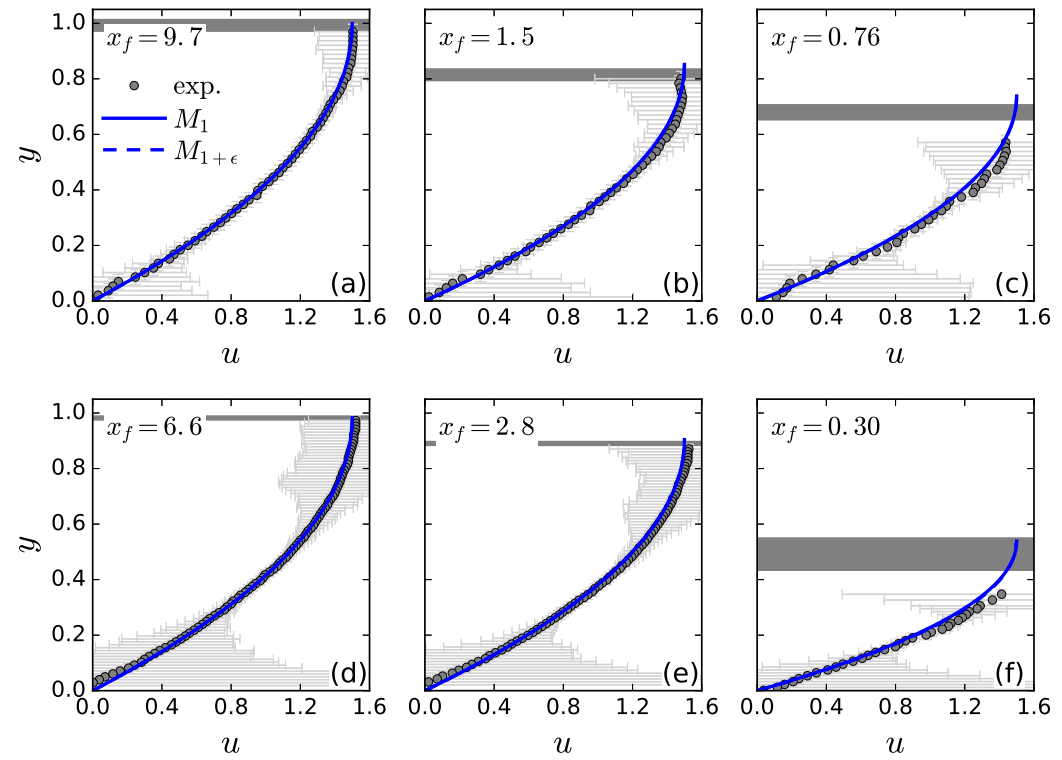

FiguRE 17. Vertical profiles of longitudinal velocity $u$ in the Newtonian case: comparison between experimental data and predictions of models $M_{1}$ and $M_{1+\epsilon}$ for different values of distance from the tip $x_{f}$ (indicated in legend). The greyed area represents experimental uncertainties on free-surface position. (a), (b), (c) $R e=0.35$ (exp. Nt1). (d), (e), (f) $R e=1.4$ (exp. Nt2).

Balmforth, N. J. \& Liu, J. J. 2004 Roll waves in mud. J. Fluid Mech. 519, 33-54.

Benney, D. J. 1966 Long waves on liquid films. J. Math. Phys. 45, 150-155.

Bernabeu, N., Saramito, P. \& Smutek, C. 2014 Numerical modeling of non-Newtonian viscoplastic flows: Part ii. Viscoplastic fluids and general tridimensional topographies. Inter. J. Numer. Anal. Model. 11, 213-228.

Bonn, D., Denn, M. M., Berthier, L., Divoux, T. \& Manneville, S. 2017 Yield stress materials in soft condensed matter. Rev. Mod. Phys. 86, 035005.

Boutounet, M., Monnier, J. \& Vila, J.-P. 2016 Multi-regime shallow free surface laminar flow models for quasi-Newtonian fluids. Eur. J. Mech. B/Fluids 55, 182-206.

Chambon, G., Ghemmour, A. \& Laigle, D. 2009 Gravity-driven surges of a viscoplastic fluid: An experimental study. J. Non-Newton. Fluid 158, 54-62.

Chambon, G., Ghemmour, A. \& NaAim, M. 2014 Experimental investigation of viscoplastic free-surface flows in steady uniform regime. J. Fluid Mech. 754, 332-364.

Chanson, N. 2004 The Hydraulics of Open Channel Flow: An Introduction, 2nd Ed.. New York: Elsevier.

Coussot, P. 1994 Steady, laminar, flow of concentrated mud suspensions in open channel. J. Hydraul. Res. 32, 535-559.

Coussot, P. 2014 Yield stress fluid flows: A review of experimental data. J. Non-Newton. Fluid 211, 31-49.

Dussan V, E. B. 1976 The moving contact line: the slip boundary condition. J. Fluid Mech. $\mathbf{7 7}, 665-684$.

Fernandez-Nieto, E. D., Noble, P. \& Vila, J. P. 2010 Shallow Water equations for nonNewtonian fluids. J. Non-Newton. Fluid 165, 712-732.

Freydier, P., Chambon, G. \& NaAim, M. 2017 Experimental characterization of velocity fields within the front of viscoplastic surges down an incline. J. Non-Newton. Fluid 240, $56-69$.

Frigand, I., Paso, K. G. \& De Souza Mendes, P. R. 2017 Bingham's model in the oil and gas industry. Rheol. Acta 56, 259-282. 
HogG, A. J. \& Pritchard, D. 2004 The effects of hydraulic resistance on dam-break and other shallow inertial flows. J. Fluid Mech. 501, 179-212.

Huang, X. \& Garcia, M. H. 1998 A Herschel-Bulkley model for mud flow down a slope. J. Fluid Mech. 374, 305-233.

Hunt, B. 1994 Newtonian fluid mechanics treatment of debris flows and avalanches. J. Hydraul. Eng. 120, 1350-1363.

Huppert, H. E. 2006 Gravity currents: a personal perspective. J. Fluid Mech. 554, 299-322.

Iverson, R. M. 2013 Mechanics of debris flows and rock avalanches. In Handbook and Environmental Fluid Dynamics (ed. H. J. S. Fernando), pp. 573-587. CRC Press / Taylor and Francis Group.

Kalliadasis, S., Ruyer-Quil, C., Scheid, B. \& Velarde, M. G. 2012 Falling Liquid Films. London: Springer.

Kirstetter, G., Hu, J., Delestre, O., Darboux, F., Lagrée, P.-Y., Popinet, S., Fullana, J. M. \& Josserand, C. 2016 Modeling rain-driven overland flow: Empirical versus analytical friction terms in the shallow water approximation. J. Hydrol. 536, 1-9.

Laigle, D. \& Coussot, P. 1997 Numerical modeling of mudflows. J. Hydraul. Eng. 123, $617-623$.

LiU, K.-F. \& MeI, C. C. 1994 Roll waves on a layer of a muddy fluid flowing down a gentle slope - A Bingham model. Phys. Fluids 6, 2577-2590.

Liu, Y., Balmforth, N. J. \& Hormozi, S. 2019 Viscoplastic surges down an incline. J. NonNewton. Fluid 268, 1-11.

Liu, Y., Balmforth, N. J., Hormozi, S. \& Hewitt, D. R. 2016 Two-dimensional viscoplastic dambreaks. J. Non-Newton. Fluid 238, 65-79.

NG, C., O \& MeI, C. C. 1994 Roll waves on a shallow layer of mud modelled as a power-law fluid. J. Fluid Mech. 263, 151-183.

Noble, P. \& Vila, J.-P. 2013 Thin power-law film flow down an inclined plane: Consistent shallow-water models and stability under large-scale perturbations. J. Fluid Mech. 735, $29-60$.

PiAU, J. M. 1996 Flow of a yield stress fluid in a long domain. Application to flow on an inclined plane. J. Rheol. 40, 711-723.

Piau, J. M. 2007 Carbopol gels: Elastoviscoplastic and slippery glasses made of individual swollen sponges. Meso- and macroscopic properties, constitutive equations and scaling laws. J. Non-Newton. Fluid 144, 1-29.

Richard, G. L., Ruyer-Quil, C. \& Vila, J. P. 2016 A three-equation model for thin films down an inclined plane. J. Fluid Mech. 804, 162-200.

Roussel, N. \& Coussot, P. 2005 "Fyfty-cent rheometer" for yield stress measurements : From slump to spreading flow. J. Rheol. 49, 705-718.

Ruyer-Quil, C., Chakraborty, S. \& Dandapat, B. S. 2012 Wavy regime of a power-law film flow. J. Fluid Mech. 692, 220-256.

Ruyer-Quil, C. \& Manneville, P. 1998 Modeling film flows down inclined planes. Eur. Phys. J. B 6, 277-292.

Ruyer-Quil, C. \& Manneville, P. 2000 Improved modeling of flows down inclined planes. Eur. Phys. J. B 15, 357-369.

Saingier, G., Deboeuf, S. \& Lagrée, P.-Y. 2016 On the front shape of an inertial granular flow down a rough incline. Phys. Fluids 28, 053302.

Saint-Venant, A. J. C. Barré DE 1871 Théorie du mouvement non permanent des eaux, avec application aux crues des rivières et à l'introduction de marées dans leurs lits. C. R. Acad. Sci. Paris 73, 147-154 and 237-240.

Savage, S. B. \& Hutter, K. 1989 The motion of a finite mass of granular material down a rough incline. J. Fluid Mech. 199, 177-215.

Schellart, W.P. 2011 Rheology and density of glucose syrup and honey: Determining their suitability for usage in analogue and fluid dynamic models of geological processes. J. Struct. Geol. 33, 1079-1088.

Whitham, G. B. 1974 Linear and Nonlinear Waves. Wiley. 